\title{
Integrability and Multisoliton Solutions of the Reverse Space or/and Time Nonlocal Fokas-Lenells (FL) Equation
}

\section{Wen-Xin Zhang}

Beijing Information Science and Technology University - Qinghe Xiaoying Campus: Beijing Information Science and Technology University

Yaqing Liu ( $\sim$ liuyaqing1981@163.com )

Beijing Information Science and Technology University https://orcid.org/0000-0001-6354-5401

\section{Research Article}

Keywords: Nonlocal Fokas-Lenells equation, Soliton solutions, Hirota bilinear method, Asymptotic analysis, Conservation laws.

Posted Date: November 5th, 2021

DOI: https://doi.org/10.21203/rs.3.rs-996855/v1

License: (c) (i) This work is licensed under a Creative Commons Attribution 4.0 International License. Read Full License

Version of Record: A version of this preprint was published at Nonlinear Dynamics on March 4th, 2022. See the published version at https://doi.org/10.1007/s11071-022-07322-9. 


\title{
Integrability and multisoliton solutions of the reverse space or/and time nonlocal Fokas-Lenells (FL) equation
}

\author{
Wen-Xin Zhang, Yaqing Liu* \\ School of Applied Science, Beijing Information Science and Technology \\ University, Beijing 100192, China
}

\begin{abstract}
This paper studies reverse space or/and time nonlocal Fokas-Lenells (FL) equation, which describes the propagation of nonlinear light pulses in monomode optical fibers when certain higher-order nonlinear effects are considered, by Hirota bilinear method. Firstly, variable transformations from reverse space nonlocal FL equation to reverse time and reverse space-time nonlocal FL equations are constructed. Secondly, the one-, two- and three-soliton solutions of the reverse space nonlocal FL equation are derived through Hirota bilinear method, and the soliton solutions of reverse time and reverse space-time nonlocal FL equations are given through variable transformations. Dynamical behaviors of the multisoliton solutions are discussed in detail by analyzing their wave structures. Thirdly, asymptotic analysis of two- and three-soliton solutions of reverse space nonlocal FL equation is used to investigated the elastic interaction and inelastic interaction. At last, the Lax integrability and conservation laws of three types of nonlocal FL equations is studied. The results obtained in this paper possess new properties that different from the ones for FL equation, which are useful in exploring novel physical phenomena of nonlocal systems in nonlinear media.
\end{abstract}

Keywords: Nonlocal Fokas-Lenells equation; Soliton solutions; Hirota bilinear method; Asymptotic analysis; Conservation laws.

\section{Introduction}

The Fokas-Lenells (FL) equation was derived as an integrable generalization of the nonlinear Schrödinger (NLS) equation using bi-Hamiltonian methods [1], which is a completely integrable nonlinear partial differential equation (here means it admits a Lax pair). The FL equation

\footnotetext{
${ }^{*}$ Corresponding author, E-mail addresses:liuyaqing1981@163.com
} 
describes the propagation of nonlinear light pulses in monomode optical fibers when certain higher-order nonlinear effects are taken into account [2,3], and it contains a lot of physical features in the solitary waves theory and optical fibers phenomena [4-6]. For constructing the soliton solutions, the bright/dark solitons and rouge waves of the FL equation, there are a large number of research literatures. The inverse scattering transformation was established by Fokas and Lenells in their original paper [2]. The theta function representations of algebrogeometric solutions were constructed in [7]. In [8] multi-Hamiltonian structure and infinitely many conservation laws were established for the vector Kaup-Newell hierarchy of the positive and negative orders. Some other methods, such as Darboux transformation method [5, 8-10], Hirota bilinear method [11-13], Riemann-Hilbert problem [14-16], Bäcklund transformation [17] and trial equation method [18] could be found in references.

Recently, the nonlocal systems have been done by Ablowitz and Musslimani when they propose the reverse space nonlocal Schrödinger equation with parity-time (PT) symmetry [19]. The solution's evolution at location $x$ depends not only on the local solution at $x$, but also on the nonlocal solution at the distant position $-x$ [20]. Since the nonlocal NLS equation was found, a large number of nonlocal integrable systems have been studied, such as nonlocal modified Korteweg-de Vries (KdV) equation [21], reverse space-time nonlocal Fokas-Lenells equation [22] and so on. Like the local case, the nonlocal systems also have integral properties, and some methods in local system are still applicable in the nonlocal systems. For instance, Gürses and Pekcan investigated the nonlocal Schrödinger equation and modified KdV equation, and found their soliton solutions by Hirota bilinear method [23, 24]. Yang et al. proposed the localized wave solutions of the reverse space nonlocal Lakshmanan-Porsezian-Daniel equation by the Darboux transformations [25]. He, Fan and Xu studied the Cauchy problem with decaying initial data for the reverse space-time nonlocal modified KdV equation by Riemann-Hilbert method [26]. Feng et al. [27] considered a nonlocal nonlinear Schrödinger equation with PT-symmetry for both zero and nonzero boundary conditions via the combination of Hirota's bilinear method and the Kadomtsev-Petviashvili hierarchy reduction method. Peng et al. [28] investigated the fully PT-symmetric inverse space nonlocal (2+1)-dimensional nonlinear NLS equation by using Hirota's bilinear method. Liu et al. [29] studied the nonlocal Gross-Pitaevskii equation with a parabolic potential employing the reduction approach on double Wronskians. The main purpose of this paper is to focus on nonlocal FL equations and research the applicatory of the Hirota bilinear method to find their multisoliton solutions. In these new types of nonlocal equations, in addition to the terms at the space-time point $(x, t)$, there are terms at the points $(-x, t),(x,-t)$ and $(-x,-t)$. It's distinctly different from local FL equation that these nonlocal FL equations have their novel spatial and/or temporal coupling, which could give rise to new physical effects and novel physical applications.

Here we consider the reverse space nonlocal Fokas-Lenells equation

$$
u_{x t}(x, t)-i u(x, t)+2 i u(x, t) u^{*}(-x, t) u_{x}(x, t)=0,
$$


where $u(x, t)$ is a complex valued function for the independent spatial variable $x$ and temporal variable $t$, and $u^{*}(-x, t)$ denotes complex conjugate of $u(x, t)$. The subscript $x$ (or $t$ ) denotes partial derivative with respect to $x$ (or $t$ ). Through the method in [20], the variable transformations from reverse space nonlocal FL equation to reverse time and reverse space-time nonlocal FL equation can be derived as follows

$$
\begin{aligned}
& \text { a) } x \rightarrow-i x, t \rightarrow i t, \\
& \text { b) } x \rightarrow-x, t \rightarrow i t .
\end{aligned}
$$

Through these variable transformations, reverse time and reverse space-time nonlocal FL equation are presented subsequently

$$
\begin{aligned}
& u_{x t}(x, t)-i u(x, t)-2 u(x, t) u^{*}(x,-t) u_{x}(x, t)=0, \\
& u_{x t}(x, t)-u(x, t)-2 u(x, t) u^{*}(-x,-t) u_{x}(x, t)=0,
\end{aligned}
$$

where $u=u(x, t)$ is a complex-valued function of $x$ and $t$, and the $*$ denotes complex conjugation. In this paper, we use the Hirota bilinear method to get one-, two- and three-soliton solutions of the reverse space nonlocal FL equation (1), then study multisoliton solutions of the reverse time and inverse space-time nonlocal FL equations through variable transforms. Asymptotic analysis is used to investigate the elastic interactions and inelastic interactions of the two solitons and the three solitons solutions, and dynamical behaviors of the multisoliton solutions are investigated by analyzing their wave structures. Finally, the Lax pairs and conservation laws of three types of nonlocal FL equations are obtained.

The outline of this paper is presented as follows. In Section 2, the one-, two- and threesoliton solutions of three types of nonlocal FL equations are obtained by using Hirota bilinear method and the variable transformations (2) and (3). And some figures are given to describe the dynamic characteristics of these soliton solutions. In Section 3, the asymptotic analysis on two- and three- soliton solutions of the reverse space nonlocal FL equation is given. In Section 4, we exhibit the Lax pairs of three types of nonlocal FL equations. Meanwhile, based on the Lax pairs, the infinitely many conservation laws of these equations (1), (4) and (5) are derived. Finally, the conclusions of this paper are stated in Section 5.

\section{Multisoliton solutions of three types of nonlocal FL e- quations}

\subsection{One-soliton solutions of three types of nonlocal FL equations}

In order to receive one-soliton solution of reverse space nonlocal FL equation, the Hirota bilinear method [30-33] and symbolic computation are used. By introducing the dependent variable 
transformations

$$
u(x, t)=\frac{G(x, t)}{F(x, t)}, u^{*}(-x, t)=\frac{G^{*}(-x, t)}{F^{*}(-x, t)},
$$

where $G(x, t), G^{*}(-x, t), F(x, t)$ and $F^{*}(-x, t)$ are complex functions, the nonlocal FL equation (1) converts into the following bilinear equation

$$
\frac{1}{F^{2}}\left(D_{x} D_{t} G \cdot F-i G F\right)+\frac{G}{F^{3}}\left(-D_{x} D_{t} F \cdot F+2 i \frac{G^{*} D_{x} G \cdot F}{F^{*}}\right)=0 .
$$

This equation can be decoupled into the following system of bilinear equations for the functions $F$ and $G$,

$$
\begin{aligned}
& D_{x} D_{t} G \cdot F=i G F \\
& D_{x} D_{t} F \cdot F=2 i \frac{G^{*} D_{x} G \cdot F}{F^{*}},
\end{aligned}
$$

where the $D_{x}$ and $D_{t}$ are bilinear operators. These operators defined as

$$
D_{x}^{m} D_{t}^{n}(G F)=\left.\left(\frac{\partial}{\partial x}-\frac{\partial}{\partial x_{1}}\right)^{m}\left(\frac{\partial}{\partial t}-\frac{\partial}{\partial t_{1}}\right)^{n} G(x, t) F\left(x_{1}, t_{1}\right)\right|_{\left(x=x_{1}, t=t_{1}\right)},
$$

where $m$ and $n$ are non-negative integers.

Solving the above series of bilinear equations (8)-(9) and combining (6), some soliton solutions can be obtained. We expand the unknown functions $(x, t), G^{*}(-x, t), F(x, t)$ and $F^{*}(-x, t)$ as a polynomial of small parameter $\epsilon$ as follows

$$
\begin{aligned}
& G(x, t)=\epsilon G_{1}+\epsilon^{3} G_{3}+\epsilon^{5} G_{5}+\cdots, \\
& G^{*}(-x, t)=\epsilon G_{1}^{*}+\epsilon^{3} G_{3}^{*}+\epsilon^{5} G_{5}^{*}+\cdots, \\
& F(x, t)=1+\epsilon^{2} F_{2}+\epsilon^{4} F_{4}+\epsilon^{6} F_{6}+\cdots, \\
& F^{*}(-x, t)=1+\epsilon^{2} F_{2}^{*}+\epsilon^{4} F_{4}^{*}+\epsilon^{6} F_{6}^{*}+\cdots,
\end{aligned}
$$

where the $G_{1}, F_{2}$, etc. are functions with spatial variable $x$ and temporal variable $t$, the functions $G_{1}^{*}, F_{2}^{*}$, etc. with variables $-x$ and $t$. Substituting the above expansions into Eqs. (8)-(9), and comparing the coefficients of $\epsilon$, the unknown functions $G(x, t), G^{*}(-x, t), F(x, t)$ and $F^{*}(-x, t)$ can be obtained by selecting appropriate functions $G_{1}, G_{1}^{*}, F_{2}, F_{2}^{*}$.

In this section, the unknown functions $G(x, t), G^{*}(-x, t), F(x, t)$ and $F^{*}(-x, t)$ are expanded in terms of a small parameter $\epsilon$ as follows

$$
\begin{aligned}
& G(x, t)=\epsilon G_{1}, \\
& G^{*}(-x, t)=\epsilon G_{1}^{*}, \\
& F(x, t)=1+\epsilon^{2} F_{2}, \\
& F^{*}(-x, t)=1+\epsilon^{2} F_{2}^{*},
\end{aligned}
$$


Substituting (12) into bilinear equation (8)-(9), we obtain a set of equations by comparing the coefficients of same powers of $\epsilon$ to zero

$$
\begin{aligned}
& G_{1 x t}=i G_{1}, \\
& F_{2 x t}=i G_{1}^{*} G_{1 x},
\end{aligned}
$$

where $G_{1}, G_{1}^{*}, F_{2}$ and $F_{2}^{*}$ are given rise to as follows

$$
\begin{aligned}
& G_{1}=e^{\eta_{1}}, \\
& G_{1}^{*}=e^{\eta_{1}^{*}}, \\
& F_{2}=A_{1} e^{\eta_{1}+\eta_{1}^{*}}, \\
& F_{2}^{*}=A_{1}^{*} e^{\eta_{1}+\eta_{1}^{*}} .
\end{aligned}
$$

We suppose that $\eta_{1}=k_{1} x-\omega_{1} t+\eta_{10}, \eta_{1}^{*}=-k_{1}^{*} x-\omega_{1}^{*} t+\eta_{10}^{*}$, and $k_{1}, k_{1}^{*}$ are arbitrary complex constants. Form Eqs. (13)-(14), the relations about $\omega_{1}, A_{1}$ and $k_{1}$ are given as follows

$$
\begin{aligned}
& \omega_{1}=-\frac{i}{k_{1}}, \\
& A_{1}=\frac{i k_{1}}{\left(k_{1}-k_{1}^{*}\right)\left(-\omega_{1}-\omega_{1}^{*}\right)} .
\end{aligned}
$$

(a)

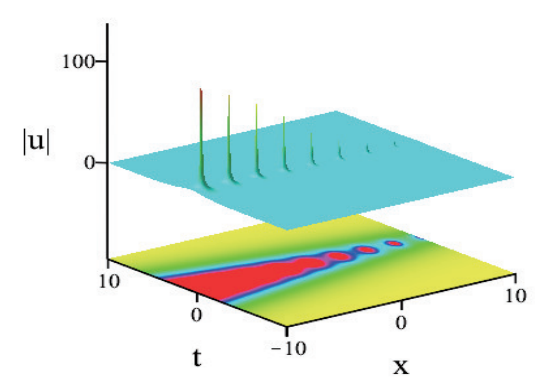

(d)

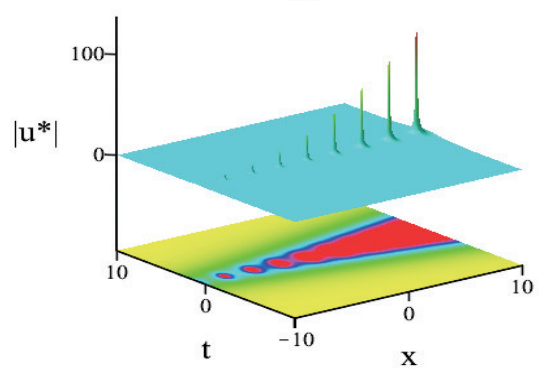

(b)

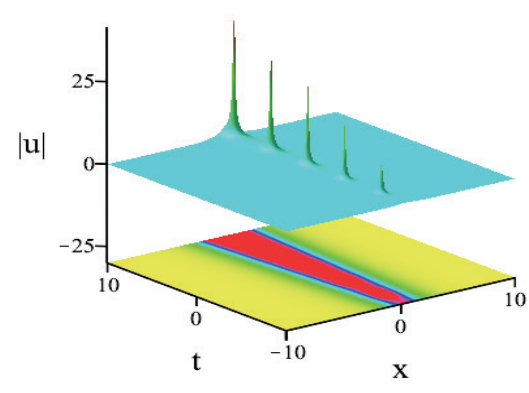

(e)

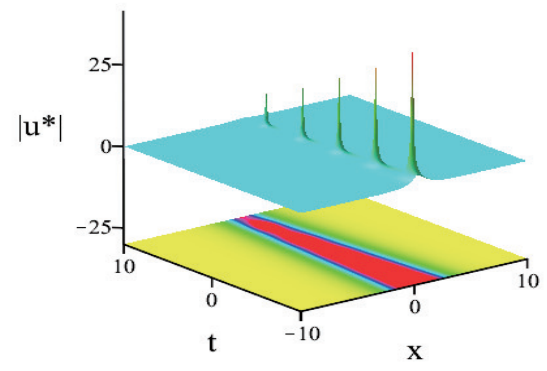

$|\mathbf{u}|$

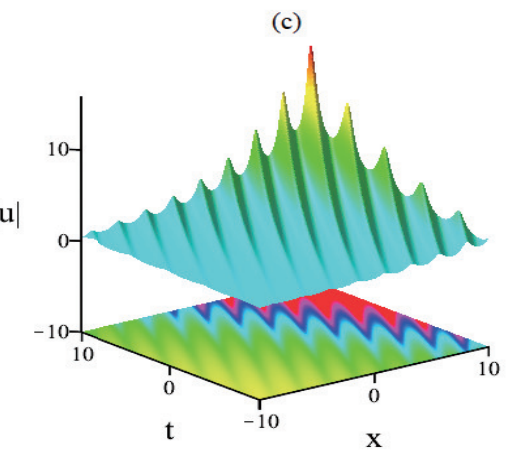

(f)

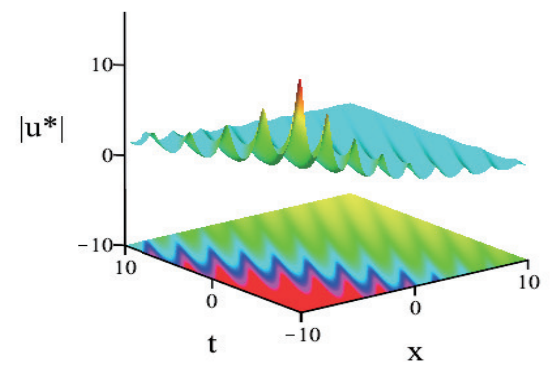

Figure 1: One soliton solutions of reverse space or/and time nonlocal FL equations (with parameters: $\left.k_{1}=-0.15+1.3 i, k_{1}^{*}=-0.15-1.3 i, \eta_{10}=\eta_{10}^{*}=0\right)$. (a) and (d) describe the reverse space FL equation; (b) and (e) describe the reverse time FL equation; (c) and (f) describe the reverse space-time FL equation. 
Since the $\omega_{1}^{*}$ is the complex conjugate of $\omega_{1}$ and the $A_{1}^{*}$ is the complex conjugate of $A_{1}$, the expressions for $\omega_{1}^{*}$ and $A_{1}^{*}$ are presented as follows

$$
\begin{aligned}
& \omega_{1}^{*}=\frac{i}{k_{1}^{*}}, \\
& A_{1}^{*}=\frac{-i k_{1}^{*}}{\left(k_{1}^{*}-k_{1}\right)\left(-\omega_{1}^{*}-\omega_{1}\right)} .
\end{aligned}
$$

Then, the general one-soliton solution of the reverse space nonlocal FL equation (1) is

$$
u(x, t)=\frac{e^{\eta_{1}}}{1+A_{1} e^{\eta_{1}+\eta_{1}^{*}}} .
$$

According to the bilinear form of parity transformed complex conjugate equation, the parity transformed complex conjugate field is derived in the form

$$
u^{*}(-x, t)=\frac{e^{\eta_{1}^{*}}}{1+A_{1}^{*} e^{\eta_{1}+\eta_{1}^{*}}} .
$$

(a)

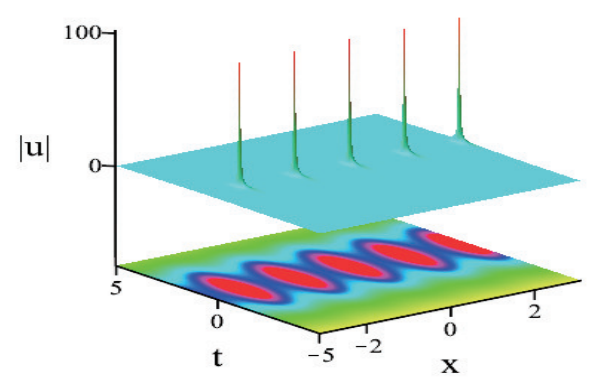

(c)

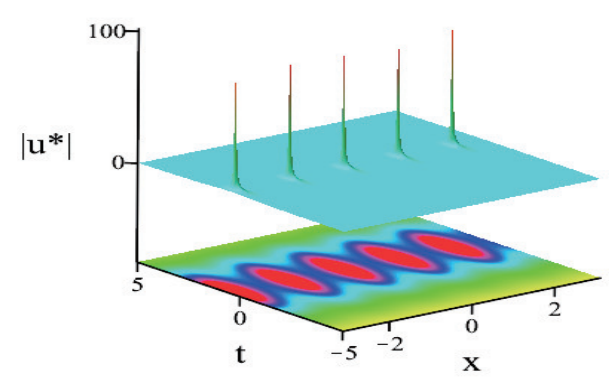

(b)

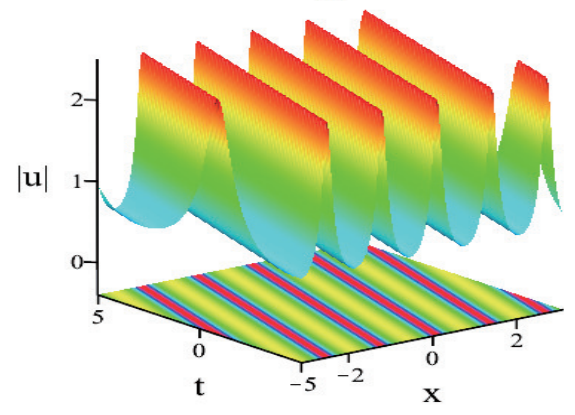

(d)

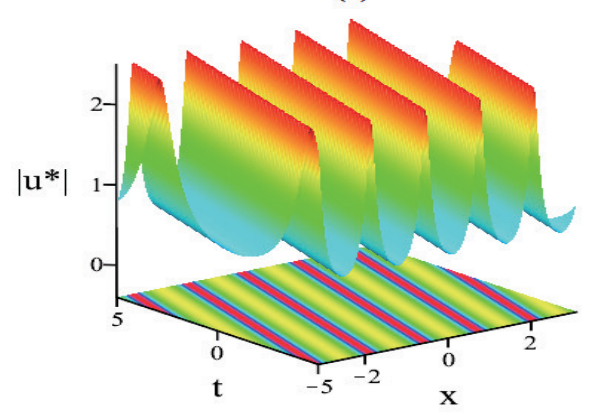

Figure 2: One soliton solutions of reverse space and reverse space-time nonlocal FL equations (with parameters: $k_{1}=2.4 i, k_{1}^{*}=-2.4 i, \eta_{10}=\eta_{10}^{*}=0$ ). (a) and (c) describe the reverse space FL equation; (b) and (d) describe the reverse space-time FL equation.

Substituting the variable transformations Eqs. (2)-(3) into one-soliton solutions Eqs. (20)(21) of the reverse space nonlocal FL equation, then one-soliton solutions of the reverse time and 
the reverse space-time nonlocal FL equation are given

$$
\begin{aligned}
& u(x, t)=\frac{e^{\xi_{1}}}{1+A_{1} e^{\xi_{1}+\xi_{1}^{*}}}, \\
& u^{*}(x,-t)=\frac{e^{\xi_{1}^{*}}}{1+A_{1}^{*} e^{\xi_{1}+\xi_{1}^{*}}}, \\
& \text { b) } u(x, t)=\frac{e^{\zeta_{1}}}{1+A_{1} e^{\zeta_{1}+\zeta_{1}^{*}}}, \\
& u^{*}(-x,-t)=\frac{e^{\zeta_{1}^{*}}}{1+A_{1}^{*} e^{\zeta_{1}+\zeta_{1}^{*}}},
\end{aligned}
$$

where $\xi_{1}=-i k_{1} x-i \omega_{1} t+\eta_{10}, \xi_{1}^{*}=i k_{1}^{*} x-i \omega_{1}^{*} t+\eta_{10}^{*}, \zeta_{1}=-k_{1} x-i \omega_{1} t+\eta_{10}, \zeta_{1}^{*}=k_{1}^{*} x-i \omega_{1}^{*} t+\eta_{10}^{*}$.

Here, some figures are provided to describe the one-soliton solutions Eqs. (20)-(25) of three types of nonlocal FL equations (see Fig.1-Fig.3). In Fig.1, (a), (b) and (c) are the profiles of $|u|$, and (d), (e) and (f) are the profiles of $\left|u^{*}\right|$. The results show that the solutions of three types of FL equations are periodic wave, and the periodic oscillations have exponential growth trend. It is obvious that $|u|$ and $\left|u^{*}\right|$ of the reverse space/time nonlocal FL equation have the same shapes as spatial/time evolution, but their enhancing shapes are antipodal. In order to intuitively observe one-soliton solutions' difference between the reverse space/time nonlocal FL equation and the reverse space-time nonlocal FL equation, more figures (Fig.2, Fig.3) are provided. These figures have the same parameters $k_{1}, k_{1}^{*}, \eta_{10}, \eta_{10}^{*}$ for different equations.

(a)

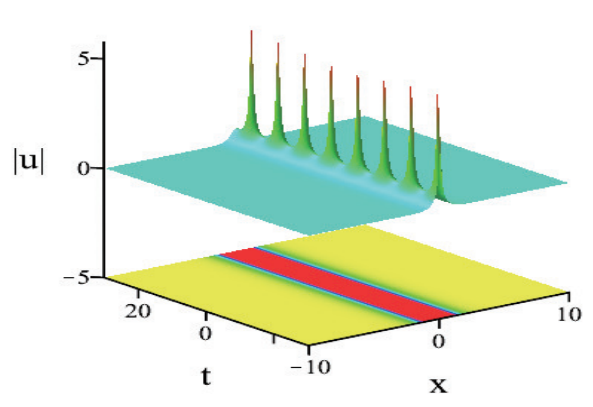

(c)

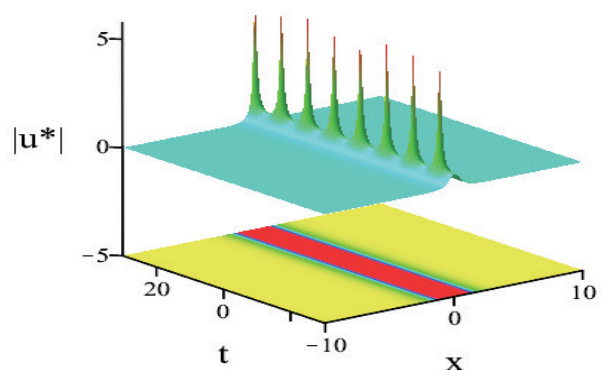

(b)

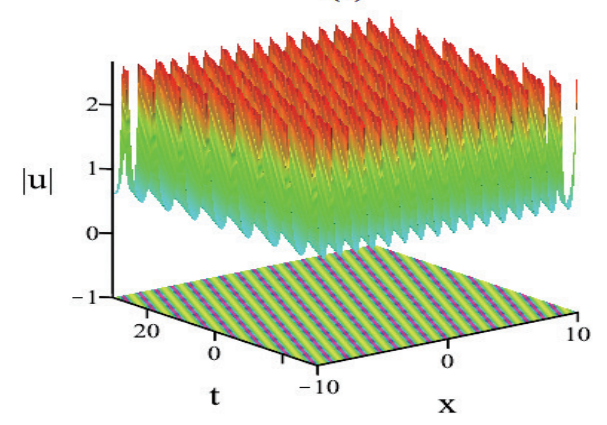

(d)

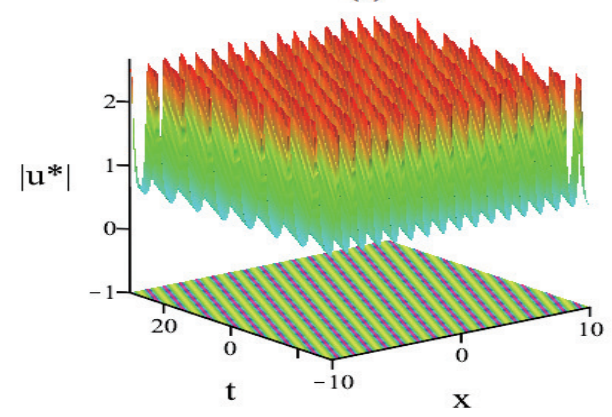

Figure 3: One soliton solutions of reverse time and reverse space-time nonlocal FL equations (with parameters: $k_{1}=2.5 i, k_{1}^{*}=-2.5 i, \eta_{10}=\eta_{10}^{*}=0$ ). (a) and (c) describe the reverse time FL equation; (b) and (d) describe the reverse space-time FL equation. 


\subsection{Two-soliton solutions of three types of nonlocal FL equations}

The two-soliton solution of the reverse space nonlocal FL equation (1) can also be obtained with Hirota bilinear method. We consider the truncating of the following expansions $G(x, t)=$ $\epsilon G_{1}+\epsilon^{3} G_{3}, G^{*}(-x, t)=\epsilon G_{1}^{*}+\epsilon^{3} G_{3}^{*}, F(x, t)=1+\epsilon^{2} F_{2}+\epsilon^{4} F_{4}, F^{*}(-x, t)=1+\epsilon^{2} F_{2}^{*}+\epsilon^{4} F_{4}^{*}$.

Substituting these expansions into the bilinear equations (8)-(9), and equating the coefficients of same powers of $\epsilon$ to zero, a set of equations can be derived

$$
\begin{aligned}
& G_{1 x t}=i G_{1}, \\
& G_{1 x t} F_{2}+G_{3 x t}-G_{1 t} F_{2 x}-G_{1 x} F_{2 t}+G_{1} F_{2 x t}=i\left(G_{1} F_{2}+G_{3}\right), \\
& F_{2 x t}=i G_{1 x} G_{1}^{*}, \\
& F_{4 x t}+F_{2} F_{2 x t}-F_{2 x} F_{2 t}+F_{2}^{*} F_{2 x t}=i G_{1}^{*}\left(G_{1 x} F_{2}+G_{3 x}-G_{1} F_{2 x}\right)+i G_{1 x} G_{3}^{*},
\end{aligned}
$$

where $G_{1}, G_{1}^{*}, F_{2}$ and $F_{2}^{*}$ are given rise to as follows

$$
\begin{aligned}
& G_{1}=e^{\eta_{1}}+e^{\eta_{2}}, \\
& G_{1}^{*}=e^{\eta_{1}^{*}}+e^{\eta_{2}^{*}}, \\
& F_{2}=A_{1} e^{\eta_{1}+\eta_{1}^{*}}+A_{2} e^{\eta_{1}+\eta_{2}^{*}}+A_{3} e^{\eta_{2}+\eta_{1}^{*}}+A_{4} e^{\eta_{2}+\eta_{2}^{*}}, \\
& F_{2}^{*}=A_{1}^{*} e^{\eta_{1}+\eta_{1}^{*}}+A_{2}^{*} e^{\eta_{1}^{*}+\eta_{2}}+A_{3}^{*} e^{\eta_{1}+\eta_{2}^{*}}+A_{4}^{*} e^{\eta_{2}+\eta_{2}^{*}} .
\end{aligned}
$$

In the above expressions, $\eta_{1}=k_{1} x-\omega_{1} t+\eta_{10}, \eta_{1}^{*}=-k_{1}^{*} x-\omega_{1}^{*} t+\eta_{10}^{*}, \eta_{2}=k_{2} x-\omega_{2} t+\eta_{20}$, $\eta_{2}^{*}=-k_{2}^{*} x-\omega_{2}^{*} t+\eta_{20}^{*}$, and $k_{1}, k_{1}^{*}, k_{2}$ and $k_{2}^{*}$ are arbitrary complex constants. Form Eqs. (26)-(28), we know

$$
\begin{aligned}
& \omega_{1}=-\frac{i}{k_{1}}, \omega_{1}^{*}=\frac{i}{k_{1}^{*}}, \\
& \omega_{2}=-\frac{i}{k_{2}}, \omega_{2}^{*}=\frac{i}{k_{2}^{*}},
\end{aligned}
$$

and

$$
\begin{aligned}
& A_{1}=\frac{i k_{1}}{\left(k_{1}-k_{1}^{*}\right)\left(-\omega_{1}-\omega_{1}^{*}\right)}, A_{1}^{*}=\frac{-i k_{1}^{*}}{\left(k_{1}^{*}-k_{1}\right)\left(-\omega_{1}^{*}-\omega_{1}\right)}, \\
& A_{2}=\frac{i k_{1}}{\left(k_{1}-k_{2}^{*}\right)\left(-\omega_{1}-\omega_{2}^{*}\right)}, A_{2}^{*}=\frac{-i k_{1}^{*}}{\left(k_{1}^{*}-k_{2}\right)\left(-\omega_{1}^{*}-\omega_{2}\right)}, \\
& A_{3}=\frac{i k_{2}}{\left(-k_{1}^{*}+k_{2}\right)\left(-\omega_{1}^{*}-\omega_{2}\right)}, A_{3}^{*}=\frac{-i k_{2}^{*}}{\left(-k_{1}+k_{2}^{*}\right)\left(-\omega_{1}-\omega_{2}^{*}\right)}, \\
& A_{4}=\frac{i k_{2}}{\left(k_{2}-k_{2}^{*}\right)\left(-\omega_{2}-\omega_{2}^{*}\right)}, A_{4}^{*}=\frac{-i k_{2}^{*}}{\left(k_{2}^{*}-k_{2}\right)\left(-\omega_{2}^{*}-\omega_{2}\right)} .
\end{aligned}
$$

Thus, a set of equations for unknown functions $G_{1}(x, t), G_{1}^{*}(-x, t), F_{2}(x, t)$ and $F_{2}^{*}(-x, t)$ are obtained. Substituting the expressions for $G_{1}$ and $F_{2}$ into the Eq. (27), the function $G_{3}$ and its parity transformed complex conjugate $G_{3}^{*}$ are given in the form

$$
\begin{aligned}
& G_{3}=B_{1} e^{\eta_{1}+\eta_{2}+\eta_{1}^{*}}+B_{2} e^{\eta_{1}+\eta_{2}+\eta_{2}^{*}}, \\
& G_{3}^{*}=B_{1}^{*} e^{\eta_{1}^{*}+\eta_{2}^{*}+\eta_{1}}+B_{2}^{*} e^{\eta_{1}^{*}+\eta_{2}^{*}+\eta_{2}},
\end{aligned}
$$


where

$$
\begin{aligned}
& B_{1}=-\frac{\left(k_{1}-k_{2}\right)^{2} k_{1}^{* 3}}{\left(k_{2}-k_{1}^{*}\right)^{2}\left(k_{1}-k_{1}^{*}\right)^{2}}, B_{2}=-\frac{\left(k_{1}-k_{2}\right)^{2} k_{2}^{* 3}}{\left(k_{2}-k_{2}^{*}\right)^{2}\left(k_{1}-k_{2}^{*}\right)^{2}}, \\
& B_{1}^{*}=-\frac{\left(k_{1}^{*}-k_{2}^{*}\right)^{2} k_{1}^{3}}{\left(k_{2}^{*}-k_{1}\right)^{2}\left(k_{1}^{*}-k_{1}\right)^{2}}, B_{2}^{*}=-\frac{\left(k_{1}^{*}-k_{2}^{*}\right)^{2} k_{2}^{3}}{\left(k_{2}^{*}-k_{2}\right)^{2}\left(k_{1}^{*}-k_{2}\right)^{2}} .
\end{aligned}
$$

Then substituting the expressions of $G_{1}, G_{1}^{*}, G_{3}, G_{3}^{*}, F_{2}$ and $F_{2}^{*}$ into Eq. (29), the functions $F_{4}$ and $F_{4}^{*}$ are derived as follows

$$
F_{4}=C_{1} e^{\eta_{1}+\eta_{2}+\eta_{1}^{*}+\eta_{2}^{*}}, F_{4}^{*}=C_{1}^{*} e^{\eta_{1}+\eta_{2}+\eta_{1}^{*}+\eta_{2}^{*}}
$$

where

$$
\begin{aligned}
C_{1} & =\frac{k_{1}^{2} k_{2}^{2}\left(k_{1}-k_{2}\right)^{2} k_{1}^{*} k_{2}^{*}\left(k_{1}^{*}-k_{2}^{*}\right)^{2}}{\left(k_{1}-k_{1}^{*}\right)^{2}\left(k_{1}-k_{2}^{*}\right)^{2}\left(k_{2}-k_{1}^{*}\right)^{2}\left(k_{2}-k_{2}^{*}\right)^{2}}, \\
C_{1}^{*} & =\frac{k_{1} k_{2}\left(k_{1}-k_{2}\right)^{2} k_{1}^{* 2} k_{2}^{* 2}\left(k_{1}^{*}-k_{2}^{*}\right)^{2}}{\left(k_{1}-k_{1}^{*}\right)^{2}\left(k_{1}-k_{2}^{*}\right)^{2}\left(k_{2}-k_{1}^{*}\right)^{2}\left(k_{2}-k_{2}^{*}\right)^{2}} .
\end{aligned}
$$

The general nonlocal two-soliton solution of the reverse space nonlocal FL equation (1) is given as follows

$$
u(x, t)=\frac{G_{1}+G_{3}}{1+F_{2}+F_{4}} .
$$

(a)

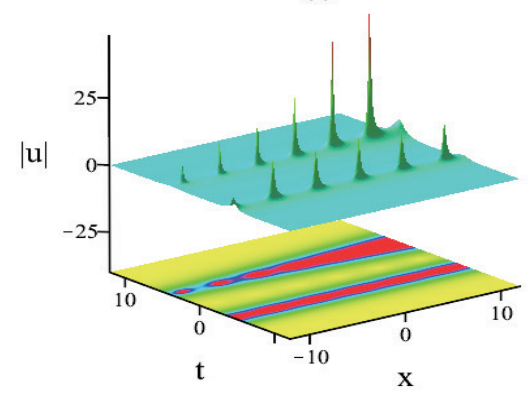

(d)

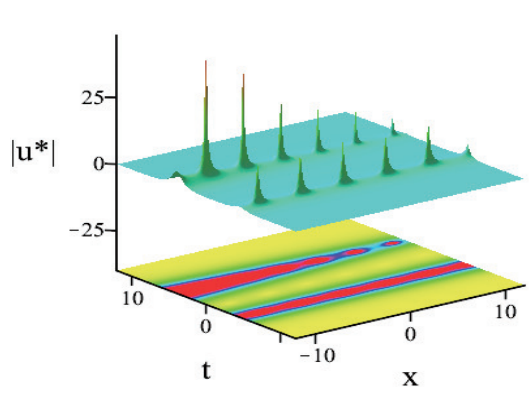

(b)

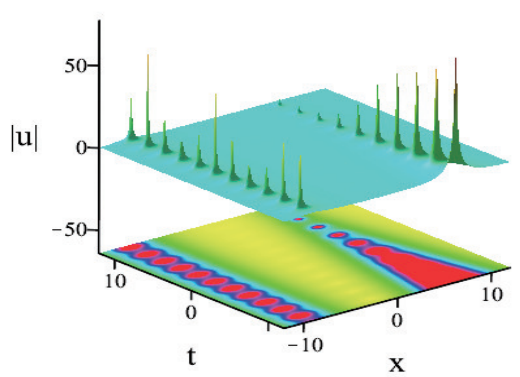

(e)

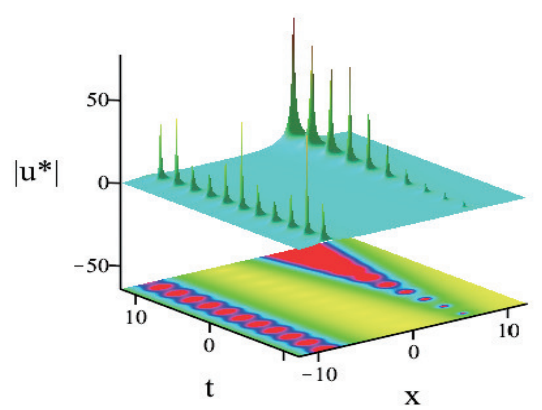

(c)
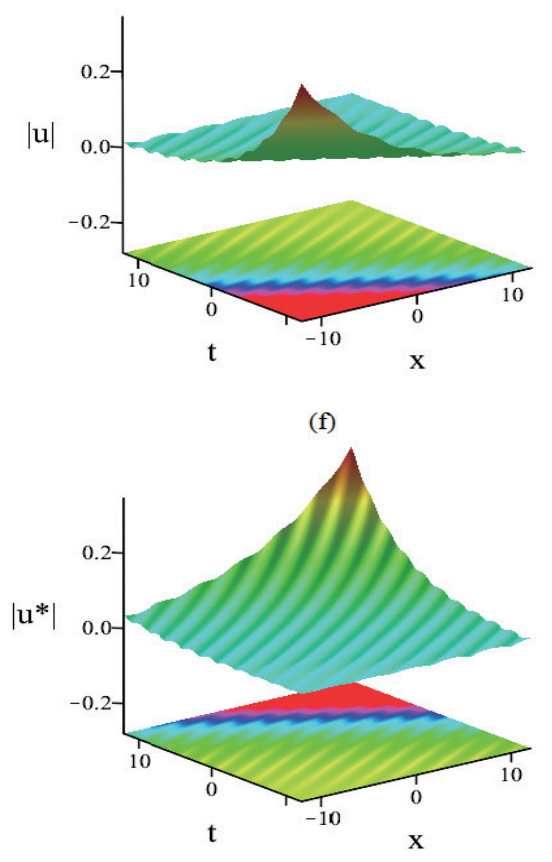

Figure 4: Two solitons solutions of reverse space or/and time nonlocal FL equations (with parameters: $\left.k_{1}=0.7 i, k_{1}^{*}=-0.7 i, k_{2}=0.1-0.8 i, k_{2}^{*}=0.1+0.8 i, \eta_{10}=\eta_{10}^{*}=2.5, \eta_{20}=\eta_{20}^{*}=1\right)$. (a) and (d) describe the reverse space FL equation; (b) and (e) describe the reverse time FL equation; (c) and (f) describe the reverse space-time FL equation. 
According to the bilinear form of parity transformed complex conjugate equation, the parity transformed complex conjugate field is derived in the form

$$
u^{*}(-x, t)=\frac{G_{1}^{*}+G_{3}^{*}}{1+F_{2}^{*}+F_{4}^{*}}
$$

Through the transformations $x=-i \hat{x}, t=i \hat{t}$ and $x=-\hat{x}, t=i \hat{t}$, the two-soliton solutions (36)-(37) of reverse space nonlocal FL equation transform into two-soliton solutions of the reverse time nonlocal FL equation (4) and the reverse space-time nonlocal FL equation (5). The solutions are presented as follows

$$
\begin{gathered}
\text { a) } u(x, t)=\frac{e^{\xi_{1}}+e^{\xi_{2}}+B_{1} e^{\xi_{1}+\xi_{2}+\xi_{1}^{*}}+B_{2} e^{\xi_{1}+\xi_{2}+\xi_{2}^{*}}}{1+A_{1} e^{\xi_{1}+\xi_{1}^{*}}+A_{2} e^{\xi_{1}+\xi_{2}^{*}}+A_{3} e^{\xi_{2}+\xi_{1}^{*}}+A_{4} e^{\xi_{2}+\xi_{2}^{*}}+C_{1} e^{\xi_{1}+\xi_{2}+\xi_{1}^{*}+\xi_{2}^{*}}}, \\
u^{*}(x,-t)=\frac{e^{\xi_{1}^{*}}+e^{\xi_{2}^{*}}+B_{1}^{*} e^{\xi_{1}^{*}+\xi_{2}^{*}+\xi_{1}}+B_{2}^{*} e^{\xi_{1}^{*}+\xi_{2}^{*}+\xi_{2}}}{1+A_{1}^{*} e^{\xi_{1}+\xi_{1}^{*}}+A_{2}^{*} e^{\xi_{1}^{*}+\xi_{2}}+A_{3}^{*} e^{\xi_{2}^{*}+\xi_{1}}+A_{4}^{*} e^{\xi_{2}+\xi_{2}^{*}}+C_{1}^{*} e^{\xi_{1}+\xi_{2}+\xi_{1}^{*}+\xi_{2}^{*}}}, \\
\text { b) } u(x, t)=\frac{e^{\zeta_{1}}+e^{\zeta_{2}}+B_{1} e^{\zeta_{1}+\zeta_{2}+\zeta_{1}^{*}}+B_{2} e^{\zeta_{1}+\zeta_{2}+\zeta_{2}^{*}}}{1+A_{1} e^{\zeta_{1}+\zeta_{1}^{*}}+A_{2} e^{\zeta_{1}+\zeta_{2}^{*}}+A_{3} e^{\zeta_{2}+\zeta_{1}^{*}}+A_{4} e^{\zeta_{2}+\zeta_{2}^{*}}+C_{1} e^{\zeta_{1}+\zeta_{2}+\zeta_{1}^{*}+\zeta_{2}^{*}}}, \\
u^{*}(-x,-t)=\frac{e^{\zeta_{1}^{*}}+e^{\zeta_{2}^{*}}+B_{1}^{*} e^{\zeta_{1}^{*}+\zeta_{2}^{*}+\zeta_{1}}+B_{2}^{*} e^{\zeta_{1}^{*}+\zeta_{2}^{*}+\zeta_{2}}}{1+A_{1}^{*} e^{\zeta_{1}+\zeta_{1}^{*}}+A_{2}^{*} e^{\zeta_{1}^{*}+\zeta_{2}}+A_{3}^{*} e^{\zeta_{2}^{*}+\zeta_{1}}+A_{4}^{*} e^{\zeta_{2}+\zeta_{2}^{*}}+C_{1}^{*} e^{\zeta_{1}+\zeta_{2}+\zeta_{1}^{*}+\zeta_{2}^{*}}},
\end{gathered}
$$

(a)

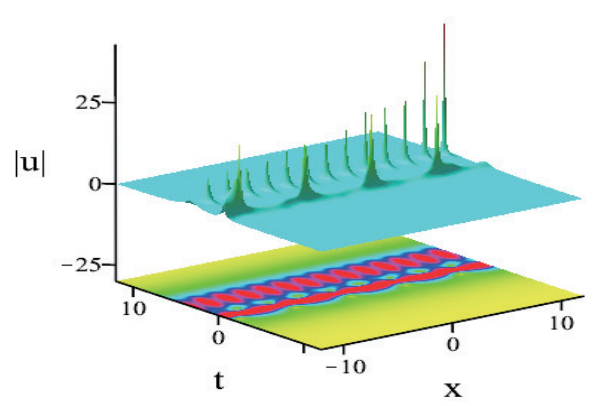

(c)

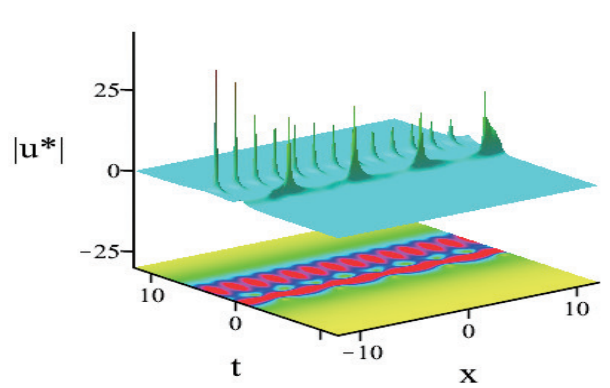

(b)

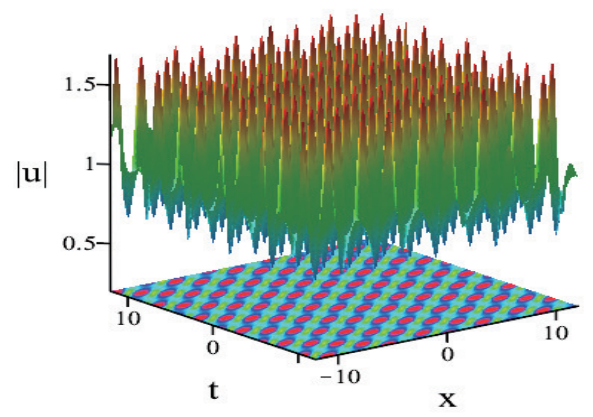

(d)

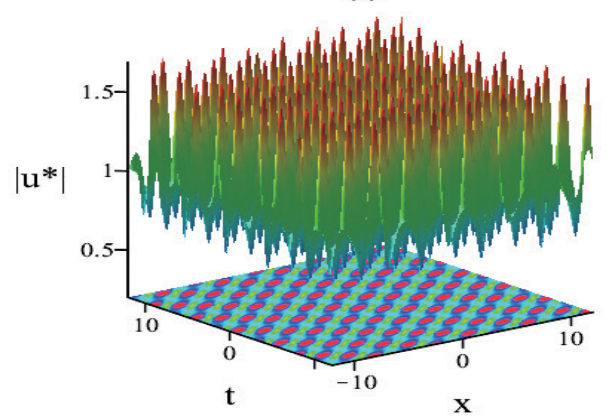

Figure 5: Two-soliton solutions of reverse space and reverse space-time nonlocal FL equations (with parameters: $k_{1}=1.74 i, k_{1}^{*}=-1.74 i, k_{2}=-0.5 i, k_{2}^{*}=0.5 i, \eta_{10}=\eta_{10}^{*}=-1.5, \eta_{20}=$ $\left.\eta_{20}^{*}=0\right)$. (a) and (c) describe the reverse space FL equation; (b) and (d) describe the reverse space-time FL equation. 
where

$$
\begin{aligned}
& \xi_{1}=-i k_{1} x-i \omega_{1} t+\eta_{10}, \xi_{1}^{*}=i k_{1}^{*} x-i \omega_{1}^{*} t+\eta_{10}^{*}, \\
& \xi_{2}=-i k_{2} x-i \omega_{2} t+\eta_{20}, \xi_{2}^{*}=i k_{2}^{*} x-i \omega_{2}^{*} t+\eta_{20}^{*}, \\
& \zeta_{1}=-k_{1} x-i \omega_{1} t+\eta_{10}, \zeta_{1}^{*}=k_{1}^{*} x-i \omega_{1}^{*} t+\eta_{10}^{*}, \\
& \zeta_{2}=-k_{2} x-i \omega_{2} t+\eta_{20}, \zeta_{2}^{*}=k_{2}^{*} x-i \omega_{2}^{*} t+\eta_{20}^{*} .
\end{aligned}
$$

(a)

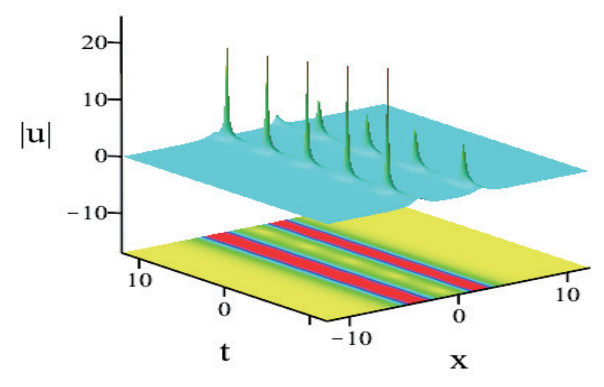

(c)

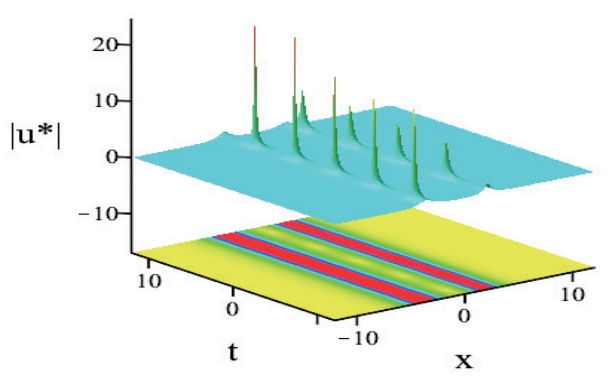

(b)

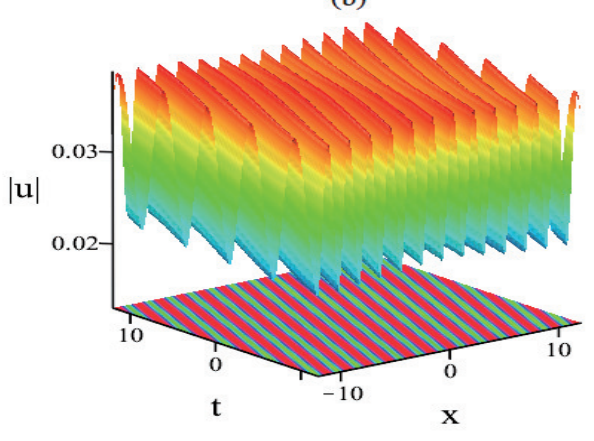

(d)

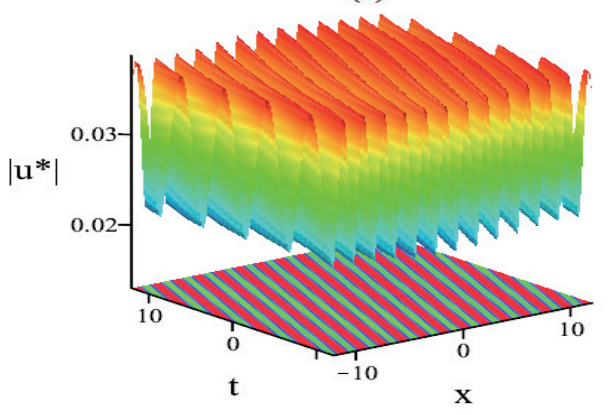

Figure 6: Two-soliton solutions of reverse time and reverse space-time nonlocal FL equations (with parameters: $k_{1}=1.5 i, k_{1}^{*}=-1.5 i, k_{2}=-1.8 i, k_{2}^{*}=1.8 i, \eta_{10}=\eta_{10}^{*}=1, \eta_{20}=\eta_{20}^{*}=-0.5$ ). (a) and (c) describe the reverse time FL equation; (b) and (d) describe the reverse space-time FL equation.

Then some figures are given to describe the two-soliton solutions (36)-(41) of three types of nonlocal FL equations (see Fig.4-Fig.6). In Fig.4, (a), (b) and (c) are the profiles of $|u|$, and (d), (e) and (f) are the profiles of $\left|u^{*}\right|$. Profiles of the reverse space nonlocal FL equation and the reverse time nonlocal FL equation present two breather-like solitons, while the two-soliton solution of the reverse space-time nonlocal FL equation are periodic wave, and the periodic oscillations have exponential growth trend. It is obvious that $|u|$ and $\left|u^{*}\right|$ of the reverse space/time FL equation have the same shapes as spatial/time evolution, but their enhancing shapes are antipodal. Figure 5 shows the comparison of the reverse space FL equation and the reverse space-time FL equation, and figure 6 shows the difference between the reverse time FL equation and the reverse space-time FL equation. These figures have the same parameters $k_{1}, k_{1}^{*}, k_{2}, k_{2}^{*}$, 
$\eta_{10}, \eta_{10}^{*}, \eta_{20}, \eta_{20}^{*}$ for different equations. Through these pictures, we could observe two-soliton solutions' differences intuitively. The shapes of two-soliton solutions of the reverse space/time FL equation are parallel with the $x$ or $t$ axis, however two-soliton solution of the reverse space-time FL equation is not parallel with neither $x$ axis nor $t$ axis, which can be viewed as a parallel superposition of time and space local solitons.

\subsection{Three-soliton solutions of three types of nonlocal FL equations}

Through Hirota bilinear method, the three-soliton solution of the reverse space nonlocal FL equation (1) can be obtained. The truncating expansions of $G(x, t), G^{*}(-x, t), F(x, t)$ and $F^{*}(-x, t)$ are given as follows

$$
\begin{aligned}
& G(x, t)=\epsilon G_{1}+\epsilon^{3} G_{3}+\epsilon^{5} G_{5}, \\
& G^{*}(-x, t)=\epsilon G_{1}^{*}+\epsilon^{3} G_{3}^{*}+\epsilon^{5} G_{5}^{*}, \\
& F(x, t)=1+\epsilon^{2} F_{2}+\epsilon^{4} F_{4}+\epsilon^{6} F_{6}, \\
& F^{*}(-x, t)=1+\epsilon^{2} F_{2}^{*}+\epsilon^{4} F_{4}^{*}+\epsilon^{6} F_{6}^{*} .
\end{aligned}
$$

Substituting these expansions into the bilinear equations (8)-(9) and equating the coefficients of same powers of $\epsilon$ to zero, a set of equations can be derived

$$
\begin{aligned}
& D_{x} D_{t} G_{1} \cdot 1=i G_{1}, \\
& D_{x} D_{t}\left(G_{1} \cdot F_{2}+G_{3} \cdot 1\right)=i\left(G_{1} F_{2}+G_{3}\right), \\
& D_{x} D_{t}\left(G_{1} \cdot F_{4}+G_{3} \cdot F_{2}+G_{5} \cdot 1\right)=i\left(G_{1} F_{4}+G_{3} F_{2}+G_{5}\right), \\
& D_{x} D_{t} 1 \cdot F_{2}=i G_{1}^{*} D_{x} G_{1} \cdot 1, \\
& D_{x} D_{t} 1 \cdot F_{4}+\frac{1}{2} D_{x} D_{t} F_{2} \cdot F_{2}+F_{2}^{*} D_{x} D_{t} 1 \cdot F_{2}=i G_{1}^{*} D_{x}\left(G_{1} \cdot F_{2}+G_{3} \cdot 1\right) \\
& +i G_{3}^{*} D_{x} G_{1} \cdot 1, \\
& D_{x} D_{t}\left(1 \cdot F_{6}+F_{2} \cdot F_{4}\right)+\frac{1}{2} F_{2}^{*} D_{x} D_{t}\left(F_{2} \cdot F_{2}\right)+F_{2}^{*} D_{x} D_{t} 1 \cdot F_{4}+F_{4}^{*} D_{x} D_{t} 1 \cdot F_{2} \\
& =i G_{1}^{*} D_{x}\left(G_{1} \cdot F_{4}+G_{3} \cdot F_{2}+G_{5} \cdot 1\right)+i G_{3}^{*} D_{x}\left(G_{1} \cdot F_{2}+G_{3} \cdot 1\right)+i G_{5}^{*} D_{x}\left(G_{1} \cdot 1\right),
\end{aligned}
$$

where $G_{1}, G_{1}^{*}, F_{2}$ and $F_{2}^{*}$ are given rise to as follows

$$
\begin{aligned}
& G_{1}=e^{\eta_{1}}+e^{\eta_{2}}+e^{\eta_{3}}, \\
& G_{1}^{*}=e^{\eta_{1}^{*}}+e^{\eta_{2}^{*}}+e^{\eta_{3}^{*}}, \\
& F_{2}=A_{1} e^{\eta_{1}+\eta_{1}^{*}}+A_{2} e^{\eta_{1}+\eta_{2}^{*}}+A_{3} e^{\eta_{2}+\eta_{1}^{*}}+A_{4} e^{\eta_{2}+\eta_{2}^{*}}+A_{5} e^{\eta_{1}+\eta_{3}^{*}}+A_{6} e^{\eta_{2}+\eta_{3}^{*}}+A_{7} e^{\eta_{3}+\eta_{1}^{*}} \\
& +A_{8} e^{\eta_{3}+\eta_{2}^{*}}+A_{9} e^{\eta_{3}+\eta_{3}^{*}}, \\
& F_{2}^{*}=A_{1}^{*} e^{\eta_{1}+\eta_{1}^{*}}+A_{2}^{*} e^{\eta_{1}^{*}+\eta_{2}}+A_{3}^{*} e^{\eta_{2}^{*}+\eta_{1}}+A_{4}^{*} e^{\eta_{2}+\eta_{2}^{*}}+A_{5}^{*} e^{\eta_{1}^{*}+\eta_{3}}+A_{6}^{*} e^{\eta_{2}^{*}+\eta_{3}}+A_{7}^{*} e^{\eta_{3}^{*}+\eta_{1}}, \\
& +A_{8}^{*} e^{\eta_{3}^{*}+\eta_{2}}+A_{9}^{*} e^{\eta 3+\eta_{3}^{*}} .
\end{aligned}
$$


In these equations, $\eta_{1}=k_{1} x-\omega_{1} t+\eta_{10}, \eta_{1}^{*}=-k_{1}^{*} x-\omega_{1}^{*} t+\eta_{10}^{*}, \eta_{2}=k_{2} x-\omega_{2} t+\eta_{20}, \eta_{2}^{*}=$ $-k_{2}^{*} x-\omega_{2}^{*} t+\eta_{20}^{*}, \eta_{3}=k_{3} x-\omega_{3} t+\eta_{30}, \eta_{3}^{*}=-k_{3}^{*} x-\omega_{3}^{*} t+\eta_{30}^{*}$, and $k_{1}, k_{2}$ and $k_{3}$ are arbitrary complex constants. Form Eqs. (42)-(45), we know

$$
\begin{aligned}
& \omega_{1}=-\frac{i}{k_{1}}, \omega_{1}^{*}=\frac{i}{k_{1}^{*}}, \\
& \omega_{2}=-\frac{i}{k_{2}}, \omega_{2}^{*}=\frac{i}{k_{2}^{*}}, \\
& \omega_{3}=-\frac{i}{k_{3}}, \omega_{3}^{*}=\frac{i}{k_{3}^{*}},
\end{aligned}
$$

and

$$
\begin{aligned}
& A_{1}=\frac{i k_{1}}{\left(k_{1}-k_{1}^{*}\right)\left(-\omega_{1}-\omega_{1}^{*}\right)}, A_{1}^{*}=\frac{-i k_{1}^{*}}{\left(k_{1}^{*}-k_{1}\right)\left(-\omega_{1}^{*}-\omega_{1}\right)}, \\
& A_{2}=\frac{i k_{1}}{\left(k_{1}-k_{2}^{*}\right)\left(-\omega_{1}-\omega_{2}^{*}\right)}, A_{2}^{*}=\frac{-i k_{1}^{*}}{\left(k_{1}^{*}-k_{2}\right)\left(-\omega_{1}^{*}-\omega_{2}\right)}, \\
& A_{3}=\frac{i k_{2}}{\left(k_{2}-k_{1}^{*}\right)\left(-\omega_{2}-\omega_{1}^{*}\right)}, A_{3}^{*}=\frac{-i k_{2}^{*}}{\left(k_{2}^{*}-k_{1}\right)\left(-\omega_{2}^{*}-\omega_{1}\right)}, \\
& A_{4}=\frac{i k_{2}}{\left(k_{2}-k_{2}^{*}\right)\left(-\omega_{2}-\omega_{2}^{*}\right)}, A_{4}^{*}=\frac{-i k_{2}^{*}}{\left(k_{2}^{*}-k_{2}\right)\left(-\omega_{2}^{*}-\omega_{2}\right)}, \\
& A_{5}=\frac{-i k_{1}^{*}}{\left(k_{1}-k_{3}^{*}\right)\left(-\omega_{1}-\omega_{3}^{*}\right)}, A_{5}^{*}=\frac{i k_{2}}{\left(k_{1}^{*}-k_{3}\right)\left(-\omega_{1}^{*}-\omega_{3}\right)}, \\
& A_{6}=\frac{-i k_{2}^{*}}{\left(k_{2}-k_{3}^{*}\right)\left(-\omega_{2}-\omega_{3}^{*}\right)}, A_{6}^{*}=\frac{i k_{3}}{\left(k_{2}^{*}-k_{3}\right)\left(-\omega_{2}^{*}-\omega_{3}\right)}, \\
& A_{7}=\frac{-i k_{3}^{*}}{\left(k_{3}-k_{1}^{*}\right)\left(-\omega_{3}-\omega_{1}^{*}\right)}, A_{7}^{*}=\frac{i k_{3}}{\left(k_{3}^{*}-k_{1}\right)\left(-\omega_{3}^{*}-\omega_{1}\right)}, \\
& A_{8}=\frac{-i k_{3}^{*}}{\left(k_{3}-k_{2}^{*}\right)\left(-\omega_{3}-\omega_{2}^{*}\right)}, A_{8}^{*}=\frac{i k_{3}}{\left(k_{3}^{*}-k_{2}\right)\left(-\omega_{3}^{*}-\omega_{2}\right)}, \\
& A_{9}=\frac{-i k_{3}^{*}}{\left(k_{3}-k_{3}^{*}\right)\left(-\omega_{3}-\omega_{3}^{*}\right)}, A_{9}^{*}=\frac{\left.1-\omega_{3}^{*}-\omega_{3}\right)}{\left(k_{3}^{*}-k_{3}\right)(}
\end{aligned}
$$

Thus, a set of equations for the unknown functions $G_{1}(x, t), G_{1}^{*}(-x, t), F_{2}(x, t)$ and $F_{2}^{*}(-x, t)$ are obtained. In order to get the function $G_{3}$ and its parity transformed complex conjugate $G_{3}^{*}$, substituting the expressions for $G_{1}$ and $F_{2}$ into Eq. (43), $G_{3}$ and $G_{3}^{*}$ are given in the form

$$
\begin{aligned}
G_{3}= & B_{1} e^{\eta_{1}+\eta_{2}+\eta_{1}^{*}}+B_{2} e^{\eta_{1}+\eta_{2}+\eta_{2}^{*}}+B_{3} e^{\eta_{1}+\eta_{2}+\eta_{3}^{*}}+B_{4} e^{\eta_{1}+\eta_{3}+\eta_{1}^{*}}+B_{5} e^{\eta_{1}+\eta_{3}+\eta_{2}^{*}} \\
& +B_{6} e^{\eta_{1}+\eta_{3}+\eta_{3}^{*}}+B_{7} e^{\eta_{2}+\eta_{3}+\eta_{1}^{*}}+B_{8} e^{\eta_{2}+\eta_{3}+\eta_{2}^{*}}+B_{9} e^{\eta_{2}+\eta_{3}+\eta_{3}^{*}}, \\
G_{3}^{*}= & B_{1}^{*} e^{\eta_{1}^{*}+\eta_{2}^{*}+\eta_{1}}+B_{2}^{*} e^{\eta_{1}^{*}+\eta_{2}^{*}+\eta_{2}}+B_{3}^{*} e^{\eta_{1}^{*}+\eta_{2}^{*}+\eta_{3}}+B_{4}^{*} e^{\eta_{1}^{*}+\eta_{3}^{*}+\eta_{1}}+B_{5}^{*} e^{\eta_{1}^{*}+\eta_{3}^{*}+\eta_{2}} \\
& +B_{6}^{*} e^{\eta_{1}^{*}+\eta_{3}^{*}+\eta_{3}}+B_{7}^{*} e^{\eta_{2}^{*}+\eta_{3}^{*}+\eta_{1}}+B_{8}^{*} e^{\eta_{2}^{*}+\eta_{3}^{*}+\eta_{2}}+B_{9}^{*} e^{\eta_{2}^{*}+\eta_{3}^{*}+\eta_{3}},
\end{aligned}
$$


where

$$
\begin{aligned}
& B_{1}=-\frac{\left(k_{1}-k_{2}\right)^{2} k_{1}^{* 3}}{\left(k_{2}-k_{1}^{*}\right)^{2}\left(k_{1}-k_{1}^{*}\right)^{2}}, B_{1}^{*}=-\frac{\left(k_{1}^{*}-k_{2}^{*}\right)^{2} k_{1}^{3}}{\left(k_{2}^{*}-k_{1}\right)^{2}\left(k_{1}^{*}-k_{1}\right)^{2}}, \\
& B_{2}=-\frac{\left(k_{1}-k_{2}\right)^{2} k_{2}^{* 3}}{\left(k_{2}-k_{2}^{*}\right)^{2}\left(k_{1}-k_{2}^{*}\right)^{2}}, B_{2}^{*}=-\frac{\left(k_{1}^{*}-k_{2}^{*}\right)^{2} k_{2}^{3}}{\left(k_{2}^{*}-k_{2}\right)^{2}\left(k_{1}^{*}-k_{2}\right)^{2}}, \\
& B_{3}=-\frac{\left(k_{1}-k_{2}\right)^{2} k_{3}^{* 3}}{\left(k_{2}-k_{3}^{*}\right)^{2}\left(k_{1}-k_{3}^{*}\right)^{2}}, B_{1}^{*}=-\frac{\left(k_{1}^{*}-k_{2}^{*}\right)^{2} k_{3}^{3}}{\left(k_{2}^{*}-k_{3}\right)^{2}\left(k_{1}^{*}-k_{3}\right)^{2}}, \\
& B_{4}=-\frac{\left(k_{1}-k_{3}\right)^{2} k_{1}^{* 3}}{\left(k_{3}-k_{1}^{*}\right)^{2}\left(k_{1}-k_{1}^{*}\right)^{2}}, B_{4}^{*}=-\frac{\left(k_{1}^{*}-k_{3}^{*}\right)^{2} k_{1}^{3}}{\left(k_{3}^{*}-k_{1}\right)^{2}\left(k_{1}^{*}-k_{1}\right)^{2}}, \\
& B_{5}=-\frac{\left(k_{1}-k_{3}\right)^{2} k_{2}^{* 3}}{\left(k_{3}-k_{2}^{*}\right)^{2}\left(k_{1}-k_{2}^{*}\right)^{2}}, B_{5}^{*}=-\frac{\left(k_{1}^{*}-k_{3}^{*}\right)^{2} k_{2}^{3}}{\left(k_{3}^{*}-k_{2}\right)^{2}\left(k_{1}^{*}-k_{2}\right)^{2}}, \\
& B_{6}=-\frac{\left(k_{1}-k_{3}\right)^{2} k_{3}^{* 3}}{\left(k_{3}-k_{3}^{*}\right)^{2}\left(k_{1}-k_{3}^{*}\right)^{2}}, B_{6}^{*}=-\frac{\left(k_{1}^{*}-k_{3}^{*}\right)^{2} k_{3}^{3}}{\left(k_{3}^{*}-k_{3}\right)^{2}\left(k_{1}^{*}-k_{3}\right)^{2}}, \\
& B_{7}=-\frac{\left(k_{2}-k_{3}\right)^{2} k_{1}^{* 3}}{\left(k_{3}-k_{1}^{*}\right)^{2}\left(k_{2}-k_{1}^{*}\right)^{2}}, B_{7}^{*}=-\frac{\left(k_{2}^{*}-k_{3}^{*}\right)^{2} k_{1}^{3}}{\left(k_{3}^{*}-k_{1}\right)^{2}\left(k_{2}^{*}-k_{1}\right)^{2}}, \\
& B_{8}=-\frac{\left(k_{2}-k_{3}\right)^{2} k_{2}^{* 3}}{\left(k_{3}-k_{2}^{*}\right)^{2}\left(k_{2}-k_{2}^{*}\right)^{2}}, B_{8}^{*}=-\frac{\left(k_{2}^{*}-k_{3}^{*}\right)^{2} k_{2}^{3}}{\left(k_{3}^{*}-k_{2}\right)^{2}\left(k_{2}^{*}-k_{2}\right)^{2}}, \\
& B_{9}=-\frac{\left(k_{2}-k_{3}\right)^{2} k_{3}^{* 3}}{\left(k_{3}-k_{3}^{*}\right)^{2}\left(k_{2}-k_{3}^{*}\right)^{2}}, B_{9}^{*}=-\frac{\left(k_{2}^{*}-k_{3}^{*}\right)^{2} k_{3}^{3}}{\left(k_{3}^{*}-k_{3}\right)^{2}\left(k_{2}^{*}-k_{3}\right)^{2}} .
\end{aligned}
$$

Then substituting the expressions of $G_{1}, G_{1}^{*}, G_{3}, G_{3}^{*}, F_{2}$ and $F_{2}^{*}$ into Eq. (46), the functions $F_{4}$ and $F_{4}^{*}$ are derived as follows

$$
\begin{aligned}
F_{4}= & C_{1} e^{\eta_{1}+\eta_{2}+\eta_{1}^{*}+\eta_{2}^{*}}+C_{2} e^{\eta_{1}+\eta_{2}+\eta_{1}^{*}+\eta_{3}^{*}}+C_{3} e^{\eta_{1}+\eta_{2}+\eta_{2}^{*}+\eta_{3}^{*}}+C_{4} e^{\eta_{1}+\eta_{3}+\eta_{1}^{*}+\eta_{2}^{*}}+ \\
& C_{5} e^{\eta_{1}+\eta_{3}+\eta_{1}^{*}+\eta_{3}^{*}}+C_{6} e^{\eta_{1}+\eta_{3}+\eta_{2}^{*}+\eta_{3}^{*}}+C_{7} e^{\eta_{2}+\eta_{3}+\eta_{1}^{*}+\eta_{2}^{*}}+C_{8} e^{\eta_{2}+\eta_{3}+\eta_{1}^{*}+\eta_{3}^{*}}+ \\
& C_{9} e^{\eta_{2}+\eta_{3}+\eta_{2}^{*}+\eta_{3}^{*}}, \\
F_{4}^{*}= & C_{1}^{*} e^{\eta_{1}^{*}+\eta_{2}^{*}+\eta_{1}+\eta_{2}}+C_{2}^{*} e^{\eta_{1}^{*}+\eta_{2}^{*}+\eta_{1}+\eta_{3}}+C_{3}^{*} e^{\eta_{1}^{*}+\eta_{2}^{*}+\eta_{2}+\eta_{3}}+C_{4}^{*} e^{\eta_{1}^{*}+\eta_{3}^{*}+\eta_{1}+\eta_{2}}+ \\
& C_{5}^{*} e^{\eta_{1}^{*}+\eta_{3}^{*}+\eta_{1}+\eta_{3}}+C_{6}^{*} e^{\eta_{1}^{*}+\eta_{3}^{*}+\eta_{2}+\eta_{3}}+C_{7}^{*} e^{\eta_{2}^{*}+\eta_{3}^{*}+\eta_{1}+\eta_{2}}+C_{8}^{*} e^{\eta_{2}^{*}+\eta_{3}^{*}+\eta_{1}+\eta_{3}}+ \\
& C_{9}^{*} e^{\eta_{2}^{*}+\eta_{3}^{*}+\eta_{2}+\eta_{3}},
\end{aligned}
$$

where

$$
\begin{aligned}
& C_{1}=\frac{\left(k_{1}^{*}-k_{2}^{*}\right)^{2}\left(k_{1}-k_{2}\right)^{2} k_{1}^{2} k_{1}^{*} k_{2}^{2} k_{2}^{*}}{\left(k_{2}-k_{2}^{*}\right)^{2}\left(k_{1}-k_{2}^{*}\right)^{2}\left(k_{2}-k_{1}^{*}\right)^{2}\left(k_{1}-k_{1}^{*}\right)^{2}}, \\
& C_{2}=\frac{\left(k_{1}^{*}-k_{3}^{*}\right)^{2}\left(k_{1}-k_{2}\right)^{2} k_{1}^{2} k_{1}^{*} k_{2}^{2} k_{3}^{*}}{\left(k_{2}-k_{3}^{*}\right)^{2}\left(k_{1}-k_{3}^{*}\right)^{2}\left(k_{2}-k_{1}^{*}\right)^{2}\left(k_{1}-k_{1}^{*}\right)^{2}}, \\
& C_{3}=\frac{\left(k_{2}^{*}-k_{3}^{*}\right)^{2}\left(k_{1}-k_{2}\right)^{2} k_{1}^{2} k_{2}^{*} k_{2}^{2} k_{3}^{*}}{\left(k_{2}-k_{3}^{*}\right)^{2}\left(k_{1}-k_{3}^{*}\right)^{2}\left(k_{2}-k_{2}^{*}\right)^{2}\left(k_{1}-k_{2}^{*}\right)^{2}}, \\
& C_{4}=\frac{\left(k_{1}^{*}-k_{2}^{*}\right)^{2}\left(k_{1}-k_{3}\right)^{2} k_{1}^{2} k_{1}^{*} k_{3}^{2} k_{2}^{*}}{\left(k_{3}-k_{2}^{*}\right)^{2}\left(k_{1}-k_{2}^{*}\right)^{2}\left(k_{3}-k_{1}^{*}\right)^{2}\left(k_{1}-k_{1}^{*}\right)^{2}},
\end{aligned}
$$




$$
\begin{aligned}
& C_{5}=\frac{\left(k_{1}^{*}-k_{3}^{*}\right)^{2}\left(k_{1}-k_{3}\right)^{2} k_{1}^{2} k_{1}^{*} k_{3}^{2} k_{3}^{*}}{\left(k_{3}-k_{3}^{*}\right)^{2}\left(k_{1}-k_{3}^{*}\right)^{2}\left(k_{3}-k_{1}^{*}\right)^{2}\left(k_{1}-k_{1}^{*}\right)^{2}}, \\
& C_{6}=\frac{\left(k_{2}^{*}-k_{3}^{*}\right)^{2}\left(k_{1}-k_{3}\right)^{2} k_{1}^{2} k_{2}^{*} k_{3}^{2} k_{3}^{*}}{\left(k_{3}-k_{3}^{*}\right)^{2}\left(k_{1}-k_{3}^{*}\right)^{2}\left(k_{3}-k_{2}^{*}\right)^{2}\left(k_{1}-k_{2}^{*}\right)^{2}}, \\
& C_{7}=\frac{\left(k_{1}^{*}-k_{2}^{*}\right)^{2}\left(k_{2}-k_{3}\right)^{2} k_{2}^{2} k_{1}^{*} k_{3}^{2} k_{2}^{*}}{\left(k_{3}-k_{2}^{*}\right)^{2}\left(k_{2}-k_{2}^{*}\right)^{2}\left(k_{3}-k_{1}^{*}\right)^{2}\left(k_{2}-k_{1}^{*}\right)^{2}}, \\
& C_{8}=\frac{\left(k_{1}^{*}-k_{3}^{*}\right)^{2}\left(k_{2}-k_{3}\right)^{2} k_{2}^{2} k_{1}^{*} k_{3}^{2} k_{3}^{*}}{\left(k_{3}-k_{3}^{*}\right)^{2}\left(k_{2}-k_{3}^{*}\right)^{2}\left(k_{3}-k_{1}^{*}\right)^{2}\left(k_{2}-k_{1}^{*}\right)^{2}}, \\
& C_{9}=\frac{\left(k_{2}^{*}-k_{3}^{*}\right)^{2}\left(k_{2}-k_{3}\right)^{2} k_{2}^{2} k_{2}^{*} k_{3}^{2} k_{3}^{*}}{\left(k_{3}-k_{3}^{*}\right)^{2}\left(k_{2}-k_{3}^{*}\right)^{2}\left(k_{3}-k_{2}^{*}\right)^{2}\left(k_{2}-k_{2}^{*}\right)^{2}},
\end{aligned}
$$

and

$$
\begin{aligned}
& C_{1}^{*}=\frac{\left(k_{1}-k_{2}\right)^{2}\left(k_{1}^{*}-k_{2}^{*}\right)^{2} k_{1}^{* 2} k_{1} k_{2}^{* 2} k_{2}}{\left(k_{2}-k_{2}^{*}\right)^{2}\left(k_{1}^{*}-k_{2}\right)^{2}\left(k_{2}^{*}-k_{1}\right)^{2}\left(k_{1}^{*}-k_{1}\right)^{2}}, \\
& C_{2}^{*}=\frac{\left(k_{1}-k_{3}\right)^{2}\left(k_{1}^{*}-k_{2}^{*}\right)^{2} k_{1}^{* 2} k_{1} k_{2}^{* 2} k_{3}}{\left(k_{2}^{*}-k_{3}\right)^{2}\left(k_{1}^{*}-k_{3}\right)^{2}\left(k_{2}^{*}-k_{1}\right)^{2}\left(k_{1}^{*}-k_{1}\right)^{2}}, \\
& C_{3}^{*}=\frac{\left(k_{2}-k_{3}\right)^{2}\left(k_{1}^{*}-k_{2}^{*}\right)^{2} k_{1}^{* 2} k_{2} k_{2}^{* 2} k_{3}}{\left(k_{2}^{*}-k_{3}\right)^{2}\left(k_{1}^{*}-k_{3}\right)^{2}\left(k_{2}^{*}-k_{2}\right)^{2}\left(k_{1}^{*}-k_{2}\right)^{2}}, \\
& C_{4}^{*}=\frac{\left(k_{1}-k_{2}\right)^{2}\left(k_{1}^{*}-k_{3}^{*}\right)^{2} k_{1}^{* 2} k_{1} k_{3}^{* 2} k_{2}}{\left(k_{3}^{*}-k_{2}\right)^{2}\left(k_{1}^{*}-k_{2}\right)^{2}\left(k_{3}^{*}-k_{1}\right)^{2}\left(k_{1}^{*}-k_{1}\right)^{2}}, \\
& C_{5}^{*}=\frac{\left(k_{1}-k_{3}\right)^{2}\left(k_{1}^{*}-k_{3}^{*}\right)^{2} k_{1}^{* 2} k_{1} k_{3}^{* 2} k_{3}}{\left(k_{3}^{*}-k_{3}\right)^{2}\left(k_{1}^{*}-k_{3}\right)^{2}\left(k_{3}^{*}-k_{1}\right)^{2}\left(k_{1}^{*}-k_{1}\right)^{2}}, \\
& C_{6}^{*}=\frac{\left(k_{2}-k_{3}\right)^{2}\left(k_{1}^{*}-k_{3}^{*}\right)^{2} k_{1}^{* 2} k_{2} k_{3}^{* 2} k_{3}}{\left(k_{3}^{*}-k_{3}\right)^{2}\left(k_{1}^{*}-k_{3}\right)^{2}\left(k_{3}^{*}-k_{2}\right)^{2}\left(k_{1}^{*}-k_{2}\right)^{2}}, \\
& C_{7}^{*}=\frac{\left(k_{1}-k_{2}\right)^{2}\left(k_{2}^{*}-k_{3}^{*}\right)^{2} k_{2}^{* 2} k_{1} k_{3}^{* 2} k_{2}}{\left(k_{3}^{*}-k_{2}\right)^{2}\left(k_{2}^{*}-k_{2}\right)^{2}\left(k_{3}^{*}-k_{1}\right)^{2}\left(k_{2}^{*}-k_{1}\right)^{2}}, \\
& C_{8}^{*}=\frac{\left(k_{1}-k_{3}\right)^{2}\left(k_{2}^{*}-k_{3}^{*}\right)^{2} k_{2}^{* 2} k_{1} k_{3}^{* 2} k_{3}}{\left(k_{3}^{*}-k_{3}\right)^{2}\left(k_{2}^{*}-k_{3}\right)^{2}\left(k_{3}^{*}-k_{1}\right)^{2}\left(k_{2}^{*}-k_{1}\right)^{2}}, \\
& C_{9}^{*}=\frac{\left(k_{2}-k_{3}\right)^{2}\left(k_{2}^{*}-k_{3}^{*}\right)^{2} k_{2}^{* 2} k_{2} k_{3}^{* 2} k_{3}}{\left(k_{3}^{*}-k_{3}\right)^{2}\left(k_{2}^{*}-k_{3}\right)^{2}\left(k_{3}^{*}-k_{2}\right)^{2}\left(k_{2}^{*}-k_{2}\right)^{2}} .
\end{aligned}
$$

So as to derive the expression of $G_{5}$, we substitute the expressions for $G_{1}, G_{1}^{*}, G_{3}, G_{3}^{*}, F_{2}$, $F_{2}^{*}, F_{4}$ and $F_{4}^{*}$ into Eq. (44), the functions $G_{5}$ and $G_{5}^{*}$ are given as follows

$$
\begin{aligned}
& G_{5}=D_{1} e^{\eta_{1}+\eta_{2}+\eta_{3}+\eta_{1}^{*}+\eta_{2}^{*}}+D_{2} e^{\eta_{1}+\eta_{2}+\eta_{3}+\eta_{1}^{*}+\eta_{3}^{*}}+D_{3} e^{\eta_{1}+\eta_{2}+\eta_{3}+\eta_{2}^{*}+\eta_{3}^{*}}, \\
& G_{5}^{*}=D_{1}^{*} e^{\eta_{1}^{*}+\eta_{2}^{*}+\eta_{3}^{*}+\eta_{1}+\eta_{2}}+D_{2}^{*} e^{\eta_{1}^{*}+\eta_{2}^{*}+\eta_{3}^{*}+\eta_{1}+\eta_{3}}+D_{3}^{*} e^{\eta_{1}^{*}+\eta_{2}^{*}+\eta_{3}^{*}+\eta_{2}+\eta_{3}},
\end{aligned}
$$

where

$$
\begin{aligned}
D_{1} & =\frac{\left(k_{1}^{*}-k_{2}^{*}\right)^{2}\left(k_{2}-k_{3}\right)^{2}\left(k_{1}-k_{3}\right)^{2}\left(k_{1}-k_{2}\right)^{2} k_{2}^{* 3} k_{1}^{* 3}}{\left(k_{2}-k_{1}^{*}\right)^{2}\left(k_{2}-k_{2}^{*}\right)^{2}\left(k_{3}-k_{2}^{*}\right)^{2}\left(k_{1}-k_{1}^{*}\right)^{2}\left(k_{3}-k_{1}^{*}\right)^{2}\left(k_{1}-k_{2}^{*}\right)^{2}}, \\
D_{2} & =\frac{\left(k_{1}^{*}-k_{3}^{*}\right)^{2}\left(k_{2}-k_{3}\right)^{2}\left(k_{1}-k_{3}\right)^{2}\left(k_{1}-k_{2}\right)^{2} k_{3}^{* 3} k_{1}^{* 3}}{\left(k_{3}-k_{1}^{*}\right)^{2}\left(k_{3}-k_{3}^{*}\right)^{2}\left(k_{2}-k_{3}^{*}\right)^{2}\left(k_{1}-k_{1}^{*}\right)^{2}\left(k_{2}-k_{1}^{*}\right)^{2}\left(k_{1}-k_{3}^{*}\right)^{2}}, \\
D_{3} & =\frac{\left(k_{2}^{*}-k_{3}^{*}\right)^{2}\left(k_{2}-k_{3}\right)^{2}\left(k_{1}-k_{3}\right)^{2}\left(k_{1}-k_{2}\right)^{2} k_{3}^{* 3} k_{2}^{* 3}}{\left(k_{3}-k_{2}^{*}\right)^{2}\left(k_{3}-k_{3}^{*}\right)^{2}\left(k_{2}-k_{3}^{*}\right)^{2}\left(k_{1}-k_{2}^{*}\right)^{2}\left(k_{2}-k_{2}^{*}\right)^{2}\left(k_{1}-k_{3}^{*}\right)^{2}},
\end{aligned}
$$


and

$$
\begin{aligned}
D_{1}^{*} & =\frac{\left(k_{1}-k_{2}\right)^{2}\left(k_{2}^{*}-k_{3}^{*}\right)^{2}\left(k_{1}^{*}-k_{3}^{*}\right)^{2}\left(k_{1}^{*}-k_{2}^{*}\right)^{2} k_{2}^{3} k_{1}^{3}}{\left(k_{2}^{*}-k_{1}\right)^{2}\left(k_{2}^{*}-k_{2}\right)^{2}\left(k_{3}^{*}-k_{2}\right)^{2}\left(k_{1}^{*}-k_{1}\right)^{2}\left(k_{3}^{*}-k_{1}\right)^{2}\left(k_{1}^{*}-k_{2}\right)^{2}}, \\
D_{2}^{*} & =\frac{\left(k_{1}-k_{3}\right)^{2}\left(k_{2}^{*}-k_{3}^{*}\right)^{2}\left(k_{1}^{*}-k_{3}^{*}\right)^{2}\left(k_{1}^{*}-k_{2}^{*}\right)^{2} k_{3}^{3} k_{1}^{3}}{\left(k_{3}^{*}-k_{1}\right)^{2}\left(k_{3}^{*}-k_{3}\right)^{2}\left(k_{2}^{*}-k_{3}\right)^{2}\left(k_{1}^{*}-k_{1}\right)^{2}\left(k_{2}^{*}-k_{1}\right)^{2}\left(k_{1}^{*}-k_{3}\right)^{2}}, \\
D_{3}^{*} & =\frac{\left(k_{2}-k_{3}\right)^{2}\left(k_{2}^{*}-k_{3}^{*}\right)^{2}\left(k_{1}^{*}-k_{3}^{*}\right)^{2}\left(k_{1}^{*}-k_{2}^{*}\right)^{2} k_{3}^{3} k_{2}^{3}}{\left(k_{3}^{*}-k_{2}\right)^{2}\left(k_{3}^{*}-k_{3}\right)^{2}\left(k_{2}^{*}-k_{3}\right)^{2}\left(k_{1}^{*}-k_{2}\right)^{2}\left(k_{2}^{*}-k_{2}\right)^{2}\left(k_{1}^{*}-k_{3}\right)^{2}} .
\end{aligned}
$$

Then, substituting the expressions for $G_{1}, G_{1}^{*}, G_{3}, G_{3}^{*}, G_{5}, G_{5}^{*}, F_{2}, F_{2}^{*}, F_{4}$ and $F_{4}^{*}$ into Eq. (47), the functions $F_{6}$ and $F_{6}^{*}$ are given as follows

$$
\begin{aligned}
& F_{6}=E_{1} e^{\eta_{1}+\eta_{2}+\eta_{3}+\eta_{1}^{*}+\eta_{2}^{*}+\eta_{3}^{*}}, \\
& F_{6}^{*}=E_{1}^{*} e^{\eta_{1}+\eta_{2}+\eta_{3}+\eta_{1}^{*}+\eta_{2}^{*}+\eta_{3}^{*}},
\end{aligned}
$$

where

$$
\begin{aligned}
E_{1}= & -\frac{M}{N}, \\
E_{1}^{*}= & -\frac{M^{*}}{N^{*}}, \\
M= & k_{3}^{*} k_{2}^{*} k_{1}^{*}\left(k_{2}^{*}-k_{3}^{*}\right)^{2} k_{1}^{2}\left(k_{2}-k_{3}\right)^{2}\left(k_{1}^{*}-k_{3}^{*}\right)^{2}\left(k_{1}-k_{3}\right)^{2} k_{2}^{2}\left(k_{1}-k_{2}\right)^{2}\left(k_{1}^{*}-k_{2}^{*}\right)^{2} k_{3}^{2}, \\
N= & \left(k_{1}^{*}-k_{2}\right)^{2}\left(k_{1}-k_{3}^{*}\right)^{2}\left(k_{2}^{*}-k_{3}\right)^{2}\left(k_{1}-k_{1}^{*}\right)^{2}\left(k_{2}-k_{2}^{*}\right)^{2}\left(k_{3}-k_{3}^{*}\right)^{2}\left(k_{1}-k_{2}^{*}\right)^{2}\left(k_{1}^{*}-k_{3}\right)^{2} \\
& \left(k_{2}-k_{3}^{*}\right)^{2} .
\end{aligned}
$$

The general nonlocal three-soliton solution of the reverse space nonlocal FL equation (1) is given as follows

$$
u(x, t)=\frac{G_{1}+G_{3}+G_{5}}{1+F_{2}+F_{4}+F_{6}} .
$$

According to the bilinear form of parity transformed complex conjugate equation, the parity transformed complex conjugate field is derived in the form

$$
u^{*}(-x, t)=\frac{G_{1}^{*}+G_{3}^{*}+G_{5}^{*}}{1+F_{2}^{*}+F_{4}^{*}+F_{6}^{*}} .
$$

In order to derive three-soliton solutions of the reverse time and reverse space-time nonlocal FL equation, we substitute transformations $x \rightarrow-i x, t \rightarrow i t$ into three-soliton solutions Eqs. (65)-(66) of the reverse space nonlocal FL equation. These solutions are presented as follows

$$
\begin{aligned}
& \text { a) } u(x, t)=\frac{G_{1}^{(1)}+G_{3}^{(1)}+G_{5}^{(1)}}{1+F_{2}^{(1)}+F_{4}^{(1)}+F_{6}^{(1)}}, \\
& u^{*}(x,-t)=\frac{G_{1}^{*(1)}+G_{3}^{*(1)}+G_{5}^{*(1)}}{1+F_{2}^{*(1)}+F_{4}^{*(1)}+F_{6}^{*(1)}}, \\
& \text { b) } u(x, t)=\frac{G_{1}^{(2)}+G_{3}^{(2)}+G_{5}^{(2)}}{1+F_{2}^{(2)}+F_{4}^{(2)}+F_{6}^{(2)}}, \\
& u^{*}(-x,-t)=\frac{G_{1}^{*(2)}+G_{3}^{*(2)}+G_{5}^{*(2)}}{1+F_{2}^{*(2)}+F_{4}^{*(2)}+F_{6}^{*(2)}} .
\end{aligned}
$$


(a)

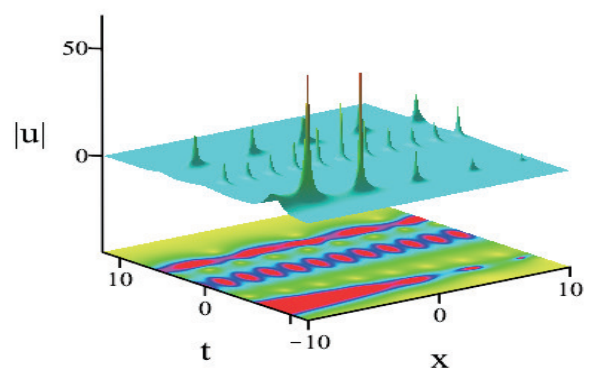

(c)

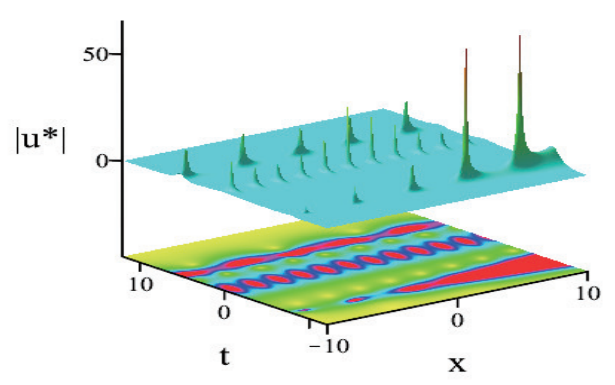

(b)

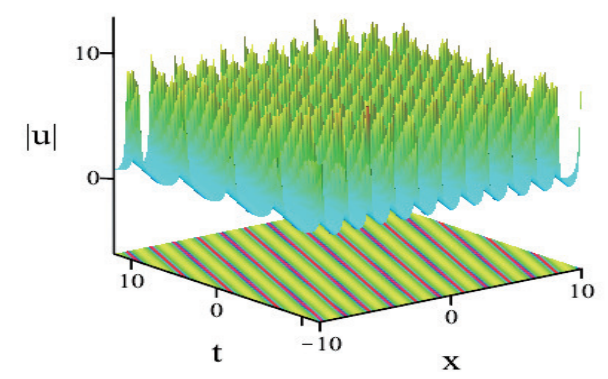

(d)

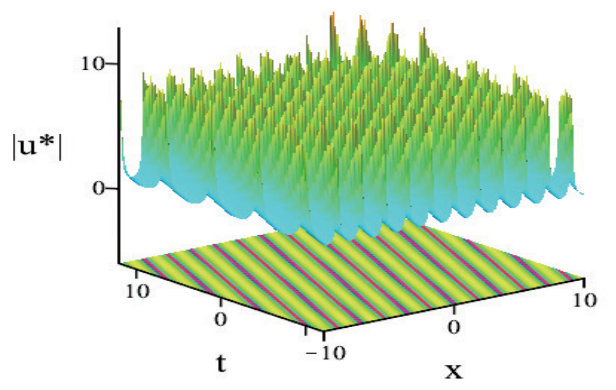

Figure 7: Three-soliton solutions of reverse space and reverse space-time nonlocal FL equations (with parameters: $k_{1}=1.75 i, k_{1}^{*}=-1.75 i, k_{2}=-0.75 i, k_{2}^{*}=0.75 i, k_{3}=-0.19-0.75 i$, $k_{3}^{*}=-0.19+0.75 i, \eta_{10}=\eta_{10}^{*}=-1.5, \eta_{20}=\eta_{20}^{*}=6, \eta_{30}=\eta_{30}^{*}=-5$ ). (a) and (c) describe the reverse space FL equation; (b) and (d) describe the reverse space-time FL equation.

where

$$
\begin{aligned}
G_{1}^{(1)}= & e^{\xi_{1}}+e^{\xi_{2}}+e^{\xi_{3}}, \\
G_{3}^{(1)}= & B_{1} e^{\xi_{1}+\xi_{2}+\xi_{1}^{*}}+B_{2} e^{\xi_{1}+\xi_{2}+\xi_{2}^{*}}+B_{3} e^{\xi_{1}+\xi_{2}+\xi_{3}^{*}}+B_{4} e^{\xi_{1}+\xi_{3}+\xi_{1}^{*}}+B_{5} e^{\xi_{1}+\xi_{3}+\xi_{2}^{*}}+ \\
& B_{6} e^{\xi_{1}+\xi_{3}+\xi_{3}^{*}}+B_{7} e^{\xi_{2}+\xi_{3}+\xi_{1}^{*}}+B_{8} e^{\xi_{2}+\xi_{3}+\xi_{2}^{*}}+B_{9} e^{\xi_{2}+\xi_{3}+\xi_{3}^{*}}, \\
G_{5}^{(1)}= & D_{1} e^{\xi_{1}+\xi_{2}+\xi_{3}+\xi_{1}^{*}+\xi_{2}^{*}}+D_{2} e^{\xi_{1}+\xi_{2}+\xi_{3}+\xi_{1}^{*}+\xi_{3}^{*}}+D_{3} e^{\xi_{1}+\xi_{2}+\xi_{3}+\xi_{2}^{*}+\xi_{3}^{*}}, \\
F_{2}^{(1)}= & A_{1} e^{\xi_{1}+\xi_{1}^{*}}+A_{2} e^{\xi_{1}+\xi_{2}^{*}}+A_{3} e^{\xi_{1}+\xi_{3}^{*}}+A_{4} e^{\xi_{2}+\xi_{1}^{*}}+A_{5} e^{\xi_{2}+\xi_{2}^{*}}+A_{6} e^{\xi_{2}+\xi_{3}^{*}}+A_{7} e^{\xi_{3}+\xi_{1}^{*}} \\
& +A_{8} e^{\xi_{3}+\xi_{2}^{*}}+A_{9} e^{\xi_{3}+\xi_{3}^{*}}, \\
F_{4}^{(1)}= & C_{1} e^{\xi_{1}+\xi_{2}+\xi_{1}^{*}+\xi_{2}^{*}}+C_{2} e^{\xi_{1}+\xi_{2}+\xi_{1}^{*}+\xi_{3}^{*}}+C_{3} e^{\xi_{1}+\xi_{2}+\xi_{2}^{*}+\xi_{3}^{*}}+C_{4} e^{\xi_{1}+\xi_{3}+\xi_{1}^{*}+\xi_{2}^{*}}+ \\
& C_{5} e^{\xi_{1}+\xi_{3}+\xi_{1}^{*}+\xi_{3}^{*}}+C_{6} e^{\xi_{1}+\xi_{3}+\xi_{2}^{*}+\xi_{3}^{*}}+C_{7} e^{\xi_{2}+\xi_{3}+\xi_{1}^{*}+\xi_{2}^{*}}+C_{8} e^{\xi_{2}+\xi_{3}+\xi_{1}^{*}+\xi_{3}^{*}}+ \\
& C_{9} e^{\xi_{2}+\xi_{3}+\xi_{2}^{*}+\xi_{3}^{*}}, \\
F_{6}^{(1)}= & E_{1} e^{\xi_{1}+\xi_{2}+\xi_{3}+\xi_{1}^{*}+\xi_{2}^{*}+\xi_{3}^{*}}, \\
\xi_{1}=- & i k_{1} x-i \omega_{1} t+\eta_{10}, \xi_{1}^{*}=i k_{1}^{*} x-i \omega_{1}^{*} t+\eta_{10}^{*}, \\
\xi_{2}=- & i k_{2} x-i \omega_{2} t+\eta_{20}, \xi_{2}^{*}=i k_{2}^{*} x-i \omega_{2}^{*} t+\eta_{20}^{*}, \\
\xi_{3}=- & i k_{3} x-i \omega_{3} t+\eta_{30}, \xi_{3}^{*}=i k_{3}^{*} x-i \omega_{3}^{*} t+\eta_{30}^{*},
\end{aligned}
$$


and

$$
\begin{aligned}
G_{1}^{(2)}= & e^{\zeta_{1}}+e^{\zeta_{2}}+e^{\zeta_{3}}, \\
G_{3}^{(2)}= & B_{1} e^{\zeta_{1}+\zeta_{2}+\zeta_{1}^{*}}+B_{2} e^{\zeta_{1}+\zeta_{2}+\zeta_{2}^{*}}+B_{3} e^{\zeta_{1}+\zeta_{2}+\zeta_{3}^{*}}+B_{4} e^{\zeta_{1}+\zeta_{3}+\zeta_{1}^{*}}+B_{5} e^{\zeta_{1}+\zeta_{3}+\zeta_{2}^{*}}+ \\
& B_{6} e^{\zeta_{1}+\zeta_{3}+\zeta_{3}^{*}}+B_{7} e^{\zeta_{2}+\zeta_{3}+\zeta_{1}^{*}}+B_{8} e^{\zeta_{2}+\zeta_{3}+\zeta_{2}^{*}}+B_{9} e^{\zeta_{2}+\zeta_{3}+\zeta_{3}^{*}}, \\
G_{5}^{(2)}= & D_{1} e^{\zeta_{1}+\zeta_{2}+\zeta_{3}+\zeta_{1}^{*}+\zeta_{2}^{*}}+D_{2} e^{\zeta_{1}+\zeta_{2}+\zeta_{3}+\zeta_{1}^{*}+\zeta_{3}^{*}}+D_{3} e^{\zeta_{1}+\zeta_{2}+\zeta_{3}+\zeta_{2}^{*}+\zeta_{3}^{*}}, \\
F_{2}^{(2)}= & A_{1} e^{\zeta_{1}+\zeta_{1}^{*}}+A_{2} e^{\zeta_{1}+\zeta_{2}^{*}}+A_{3} e^{\zeta_{1}+\zeta_{3}^{*}}+A_{4} e^{\zeta_{2}+\zeta_{1}^{*}}+A_{5} e^{\zeta_{2}+\zeta_{2}^{*}}+A_{6} e^{\zeta_{2}+\zeta_{3}^{*}}+A_{7} e^{\zeta_{3}+\zeta_{1}^{*}} \\
& +A_{8} e^{\zeta_{3}+\zeta_{2}^{*}}+A_{9} e^{\zeta_{3}+\zeta_{3}^{*}}, \\
F_{4}^{(2)}= & C_{1} e^{\zeta_{1}+\zeta_{2}+\zeta_{1}^{*}+\zeta_{2}^{*}}+C_{2} e^{\zeta_{1}+\zeta_{2}+\zeta_{1}^{*}+\zeta_{3}^{*}}+C_{3} e^{\zeta_{1}+\zeta_{2}+\zeta_{2}^{*}+\zeta_{3}^{*}}+C_{4} e^{\zeta_{1}+\zeta_{3}+\zeta_{1}^{*}+\zeta_{2}^{*}}+ \\
& C_{5} e^{\zeta_{1}+\zeta_{3}+\zeta_{1}^{*}+\zeta_{3}^{*}}+C_{6} e^{\zeta_{1}+\zeta_{3}+\zeta_{2}^{*}+\zeta_{3}^{*}}+C_{7} e^{\zeta_{2}+\zeta_{3}+\zeta_{1}^{*}+\zeta_{2}^{*}}+C_{8} e^{\zeta_{2}+\zeta_{3}+\zeta_{1}^{*}+\zeta_{3}^{*}}+ \\
& C_{9} e^{\zeta_{2}+\zeta_{3}+\zeta_{2}^{*}+\zeta_{3}^{*}}, \\
F_{6}^{(2)}= & E_{1} e^{\zeta_{1}+\zeta_{2}+\zeta_{3}+\zeta_{1}^{*}+\zeta_{2}^{*}+\zeta_{3}^{*}}, \\
\zeta_{1}=- & k_{1} x-i \omega_{1} t+\eta_{10}, \zeta_{1}^{*}=k_{1}^{*} x-i \omega_{1}^{*} t+\eta_{10}^{*}, \\
\zeta_{2}=- & k_{2} x-i \omega_{2} t+\eta_{20}, \zeta_{2}^{*}=k_{2}^{*} x-i \omega_{2}^{*} t+\eta_{20}^{*}, \\
\zeta_{3}=- & k_{3} x-i \omega_{3} t+\eta_{30}, \zeta_{3}^{*}=k_{3}^{*} x-i \omega_{3}^{*} t+\eta_{30}^{*} .
\end{aligned}
$$

(a)

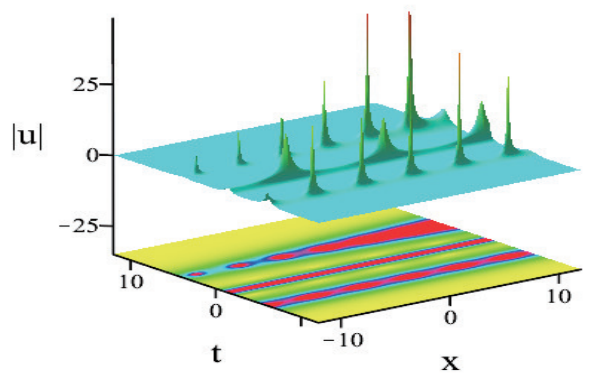

(c)

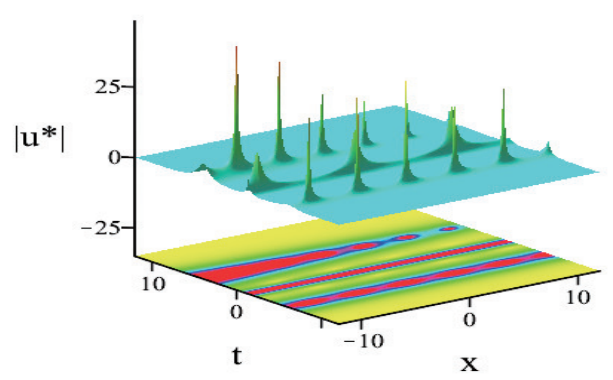

(b)

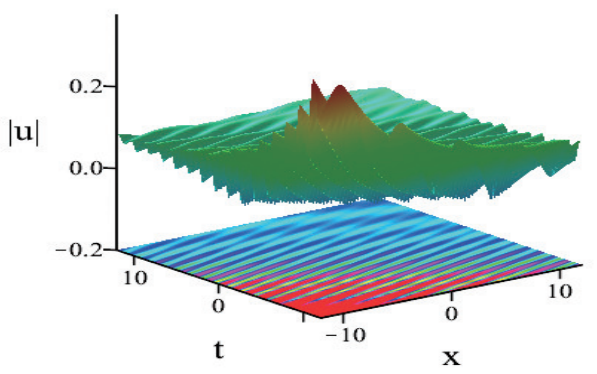

(d)

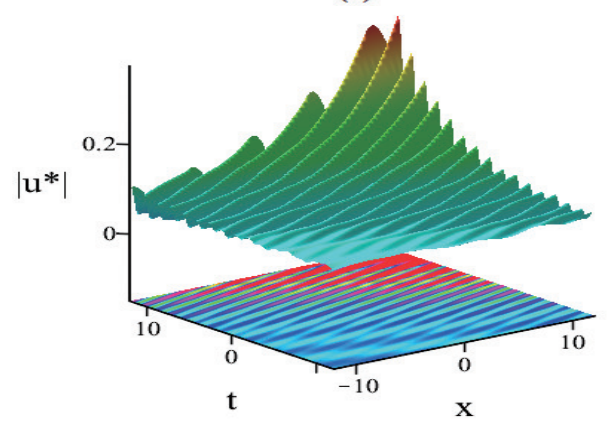

Figure 8: Three-soliton solutions of reverse space and reverse space-time nonlocal FL equations (with parameters: $k_{1}=0.7 i, k_{1}^{*}=-0.7 i, k_{2}=0.1-0.8 i, k_{2}^{*}=0.1+0.8 i, k_{3}=-0.35 i$, $k_{3}^{*}=0.35 i, \eta_{10}=\eta_{10}^{*}=2.5, \eta_{20}=\eta_{20}^{*}=1, \eta_{30}=\eta_{30}^{*}=-3$ ). (a) and (c) describe the reverse space FL equation; (b) and (d) describe the reverse space-time FL equation. 
Then some figures are presented to describe the three-soliton solutions (65)-(70) of three types of nonlocal FL equations (see Fig.7-Fig.10). In these figures, (a) and (b) are the profiles of $|u|$, (c) and (d) are the profiles of $\left|u^{*}\right|$. Figure 7 and figure 8 show the comparison between the reverse space FL equation and the reverse space-time FL equation. And figure 9 and figure 10 show the difference between the reverse time FL equation and the reverse space-time FL equation. These figures have the same parameters $k_{1}, k_{2}, k_{3}, \eta_{10}, \eta_{20}$ and $\eta_{30}$ for different equations. Through these pictures, the difference between three-soliton solutions of two different nonlocal FL equations can be observed intuitively. It is obvious that $|u|$ and $\left|u^{*}\right|$ of the reverse space/time FL equation have the same shapes as spatial/time evolution, but their enhancing shapes are antipodal, and profiles of the reverse space FL equation and the reverse time FL equation present three breather-like solitons. The solutions of the reverse space-time FL equation are periodic, and in Fig.8 and Fig.10 the results exhibit the periodic oscillations with exponential growth trend, and $|u(x, t)|$ and $\left|u^{*}(-x,-t)\right|$ have the opposite enhancing directions as time evolution. Through these figures, the shapes of three-soliton solutions of the reverse space/time FL equation are parallel with the $x$ or $t$ axis, however three-soliton solution of the reverse space-time FL equation can be viewed the parallel superposition of time and space local breather-like solitons.

(a)

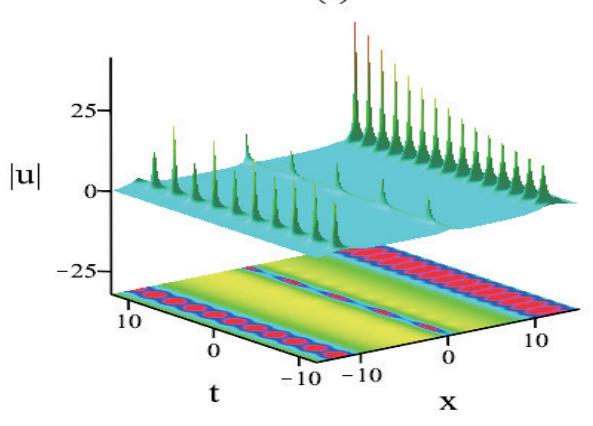

(c)

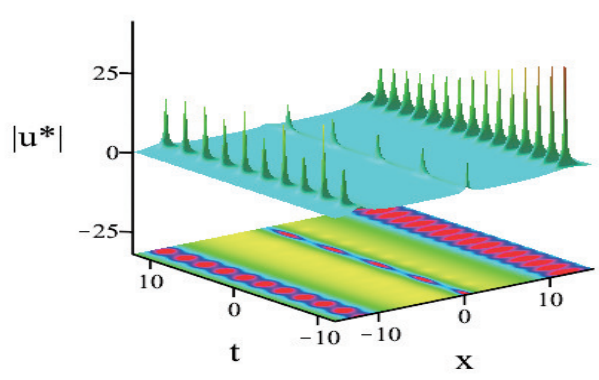

(b)

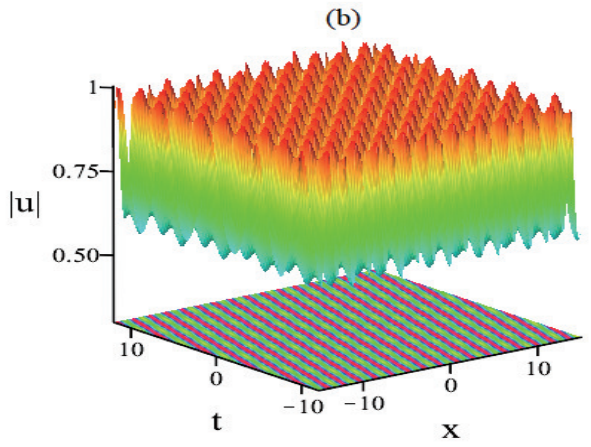

(d)

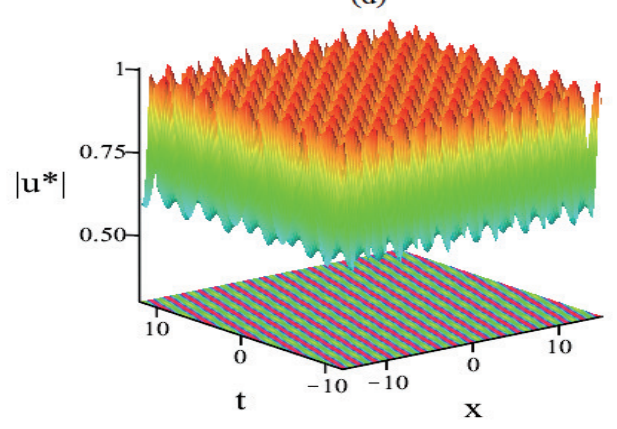

Figure 9: Three-soliton solutions of reverse time and reverse space-time nonlocal FL equations (with parameters: $k_{1}=1.7 i, k_{1}^{*}=-1.7 i, k_{2}=-0.5 i, k_{2}^{*}=0.5 i, k_{3}=-0.75 i, k_{3}^{*}=0.75 i$, $\eta_{10}=\eta_{10}^{*}=-1.5, \eta_{20}=\eta_{20}^{*}=6, \eta_{30}=\eta_{30}^{*}=-5$ ). (a) and (c) describe the reverse time FL equation; (b) and (d) describe the reverse space-time FL equation. 
(a)

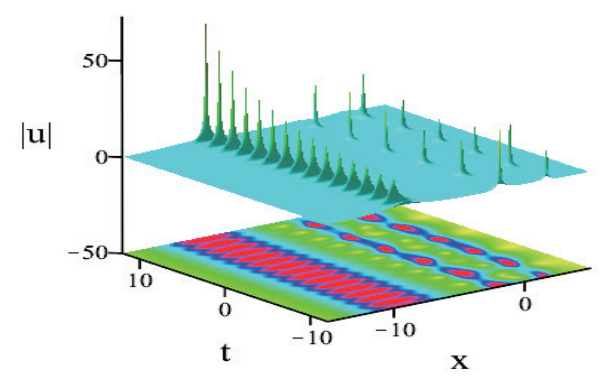

(c)

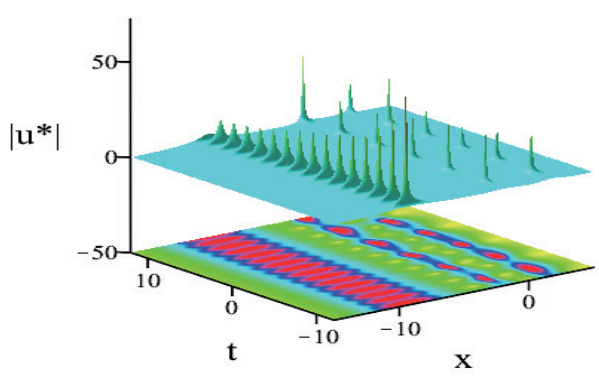

(b)

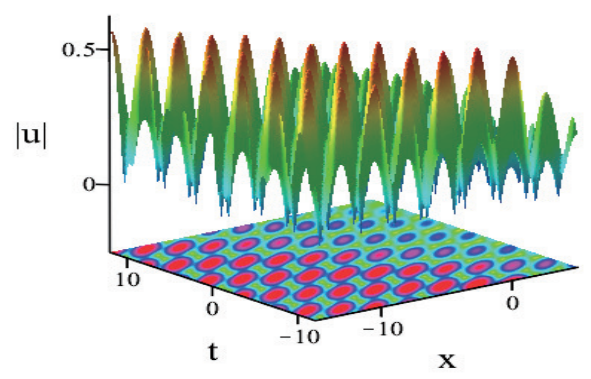

(d)

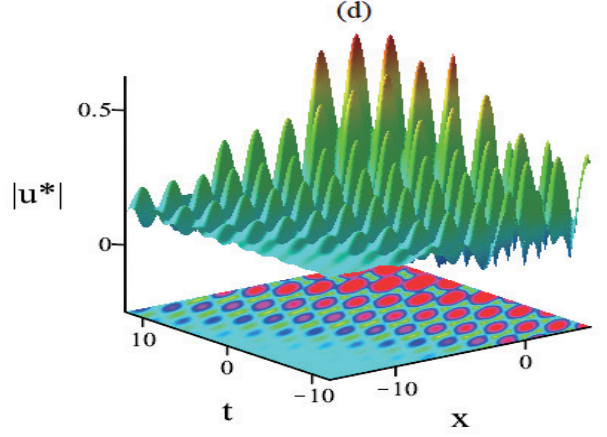

Figure 10: Three solitons solutions of reverse time and reverse space-time nonlocal FL equations (with parameters: $k_{1}=0.5 i, k_{1}^{*}=-0.5 i, k_{2}=0.25-1.5 i, k_{2}^{*}=0.25+1.5 i, k_{3}=-1.22 i$, $k_{3}^{*}=1.22 i, \eta_{10}=\eta_{10}^{*}=2.5, \eta_{20}=\eta_{20}^{*}=1, \eta_{30}=\eta_{30}^{*}=1$ ). (a) and (c) describe the reverse time FL equation; (b) and (d) describe the reverse space-time FL equation.

\section{$3 \quad$ Asymptotic analysis}

\subsection{Asymptotic analysis on two-soliton solution of the reverse space FL equation}

Through asymptotic analysis in [34], it shows that when solitons undergo multiple collisions, there exists possibility of soliton's shape restoration. Asymptotic analysis is used to investigate the elastic and inelastic interactions between the bound solitons and the regular one soliton [35].

Considering the above two-soliton solution Eq. (36), without loss of generality, we assume that $\eta_{10}=\eta_{20}=0$ and $k_{1} / k_{2}>0$. For fixed $\eta_{1}$, note that $\eta_{2}+\eta_{2}^{*}=2 \operatorname{Re}\left(\frac{k_{2}}{k_{1}} \eta_{1}\right)+2 \operatorname{Re}\left(\frac{k_{2}}{k_{1}} \omega_{1}-\omega_{2}\right) t$, and suppose $\operatorname{Re}\left(\frac{k_{2}}{k_{1}} \omega_{1}-\omega_{2}\right)>0$.

i) Taking limit $t \rightarrow-\infty: \eta_{1}+\eta_{1}^{*} \sim 0, \eta_{2}+\eta_{2}^{*} \sim-\infty$, the asymptotic expressions for the two solitons before interaction can be given by

$$
\begin{aligned}
u^{1-} & \sim \frac{1}{2} e^{\frac{\eta_{1}-\eta_{1}^{*}-\alpha_{1}}{2}} \operatorname{sech}\left(\frac{\eta_{1}+\eta_{1}^{*}+\alpha_{1}}{2}\right), \\
e^{\alpha_{1}} & =A_{1}=\frac{i k_{1}}{\left(k_{1}-k_{1}^{*}\right)\left(-\omega_{1}-\omega_{1}^{*}\right)} .
\end{aligned}
$$


ii) Taking limit $t \rightarrow+\infty: \eta_{1}+\eta_{1}^{*} \sim 0, \eta_{2}+\eta_{2}^{*} \sim+\infty$, the asymptotic expressions for the two solitons after interaction can be given by

$$
\begin{aligned}
u^{1+} & \sim \frac{B_{2}}{2 A_{4}} e^{\frac{\eta_{1}-\eta_{1}^{*}-\alpha_{2}}{2}} \operatorname{sech}\left(\frac{\eta_{1}+\eta_{1}^{*}+\alpha_{2}}{2}\right) \\
e^{\alpha_{2}} & =\frac{C_{1}}{A_{4}}=-\frac{\left(k_{1}-k_{2}\right)^{2}\left(k_{1}^{*}-k_{2}^{*}\right)^{2} k_{1}^{2} k_{1}^{*}}{\left(k_{1}-k_{1}^{*}\right)^{2}\left(k_{1}-k_{2}^{*}\right)^{2}\left(k_{2}-k_{1}^{*}\right)^{2}} .
\end{aligned}
$$

For fixed $\eta_{2}$, note that $\eta_{1}+\eta_{1}^{*}=2 \operatorname{Re}\left(\frac{k_{1}}{k_{2}} \eta_{2}\right)+2 \operatorname{Re}\left(\frac{k_{1}}{k_{2}} \omega_{2}-\omega_{1}\right) t$, and it is obvious that $\operatorname{Re}\left(\frac{k_{1}}{k_{2}} \omega_{2}-\right.$ $\left.\omega_{1}\right)<0$.

i) Taking limit $t \rightarrow-\infty: \eta_{1}+\eta_{1}^{*} \sim+\infty, \eta_{2}+\eta_{2}^{*} \sim 0$, the asymptotic expressions for the two solitons before interaction can be given by

$$
\begin{aligned}
u^{2-} & \sim \frac{B_{1}}{2 A_{1}} e^{\frac{\eta_{2}-\eta_{2}^{*}-\alpha_{3}}{2}} \operatorname{sech}\left(\frac{\eta_{2}+\eta_{2}^{*}+\alpha_{3}}{2}\right) \\
e^{\alpha_{3}} & =\frac{C_{1}}{A_{1}}=-\frac{\left(k_{1}-k_{2}\right)^{2}\left(k_{1}^{*}-k_{2}^{*}\right)^{2} k_{2}^{2} k_{2}^{*}}{\left(k_{1}-k_{2}^{*}\right)^{2}\left(k_{2}-k_{1}^{*}\right)^{2}\left(k_{2}-k_{2}^{*}\right)^{2}} .
\end{aligned}
$$

ii) Taking limit $t \rightarrow+\infty: \eta_{1}+\eta_{1}^{*} \sim-\infty, \eta_{2}+\eta_{2}^{*} \sim 0$, the asymptotic expressions for the two solitons after interaction can be given by

$$
\begin{aligned}
u^{2+} & \sim \frac{1}{2} e^{\frac{\eta_{2}-\eta_{2}^{*}-\alpha_{4}}{2}} \operatorname{sech}\left(\frac{\eta_{2}+\eta_{2}^{*}+\alpha_{4}}{2}\right), \\
e^{\alpha_{4}} & =A_{4}=\frac{i k_{2}}{\left(k_{2}-k_{2}^{*}\right)\left(-\omega_{2}-\omega_{2}^{*}\right)} .
\end{aligned}
$$

Comparing the asymptotic expressions of two-soliton solution between before interaction and after interaction, we find that $k_{1}, k_{1}^{*}, k_{2}$ and $k_{2}^{*}$ accord with the conditions

$$
\frac{k_{1}^{2}\left|k_{2}-k_{1}^{*}\right|\left|k_{1}^{*}-k_{2}^{*}\right|}{k_{1}^{* 2}\left|k_{1}-k_{2}\right|\left|k_{1}-k_{2}^{*}\right|}=1 \text { and } \frac{k_{2}^{2}\left|k_{1}-k_{2}^{*}\right|\left|k_{1}^{*}-k_{2}^{*}\right|}{k_{2}^{* 2}\left|k_{1}-k_{2}\right|\left|k_{2}-k_{1}^{*}\right|}=1 \text {, }
$$

the relations of amplitudes can be obtained

$$
\mathrm{Am}^{1-}=\mathrm{Am}^{1+} \text { and } \mathrm{Am}^{2-}=\mathrm{Am}^{2+},
$$

where $\mathrm{Am}^{1-}$ and $\mathrm{Am}^{2-}$ denote the amplitudes for the two solitons before the interaction, while $\mathrm{Am}^{1+}$ and $\mathrm{Am}^{2+}$ denote the amplitudes for the two solitons after the interaction. When $k_{1}, k_{1}^{*}$, $k_{2}$ and $k_{2}^{*}$ do not accord with conditions $(75)$, it can be yields

$$
\mathrm{Am}^{1-} \neq \mathrm{Am}^{1+} \text { and } \mathrm{Am}^{2-} \neq \mathrm{Am}^{2+}
$$

Through expressions (76) and (77), it is obvious that the elastic interaction for two-soliton solution of the reverse space nonlocal FL equation appears under conditions (75), inelastic interaction for two-soliton solution of the reverse space nonlocal FL equation arises beyond conditions $(75)$. 


\subsection{Asymptotic analysis on three-soliton solution of the reverse space FL equation}

Considering the above three-soliton solution Eq. (65), without loss of generality, we assume that $\eta_{10}=\eta_{20}=\eta_{30}=0, k_{1} / k_{2}>0, k_{2} / k_{3}>0$ and $k_{1} / k_{3}>0$. For fixed $\eta_{1}$, note that $\eta_{2}+\eta_{2}^{*}=2 \operatorname{Re}\left(\frac{k_{2}}{k_{1}} \eta_{1}\right)+2 \operatorname{Re}\left(\frac{k_{2}}{k_{1}} \omega_{1}-\omega_{2}\right) t$ and $\eta_{3}+\eta_{3}^{*}=2 \operatorname{Re}\left(\frac{k_{3}}{k_{1}} \eta_{1}\right)+2 \operatorname{Re}\left(\frac{k_{3}}{k_{1}} \omega_{1}-\omega_{3}\right) t$, and suppose $\operatorname{Re}\left(\frac{k_{2}}{k_{1}} \omega_{1}-\omega_{2}\right)>0$ and $\operatorname{Re}\left(\frac{k_{3}}{k_{1}} \omega_{1}-\omega_{3}\right)>0$.

i) Taking limit $t \rightarrow-\infty: \eta_{1}+\eta_{1}^{*} \sim 0, \eta_{2}+\eta_{2}^{*} \sim-\infty, \eta_{3}+\eta_{3}^{*} \sim-\infty$, the asymptotic expressions for the three solitons before interaction can be given by

$$
\begin{aligned}
u^{1-} & \sim \frac{1}{2} e^{\frac{\eta_{1}-\eta_{1}^{*}-\alpha_{1}}{2}} \operatorname{sech}\left(\frac{\eta_{1}+\eta_{1}^{*}+\alpha_{1}}{2}\right), \\
e^{\alpha_{1}} & =A_{1}=\frac{i k_{1}}{\left(k_{1}-k_{1}^{*}\right)\left(-\omega_{1}-\omega_{1}^{*}\right)} .
\end{aligned}
$$

ii) Taking limit $t \rightarrow+\infty: \eta_{1}+\eta_{1}^{*} \sim 0, \eta_{2}+\eta_{2}^{*} \sim+\infty, \eta_{3}+\eta_{3}^{*} \sim+\infty$, the asymptotic expressions for the three solitons after interaction can be given by

$$
\begin{aligned}
& u^{1+} \sim \frac{D_{3}}{2 C_{9}} e^{\frac{\eta_{1}-\eta_{1}^{*}-\alpha_{5}}{2}} \operatorname{sech}\left(\frac{\eta_{1}+\eta_{1}^{*}+\alpha_{5}}{2}\right), \\
& e^{\alpha_{5}}=\frac{E_{1}}{C_{9}}=-\frac{\left(k_{1}-k_{2}\right)^{2}\left(k_{1}-k_{3}\right)^{2}\left(k_{1}^{*}-k_{2}^{*}\right)^{2}\left(k_{1}^{*}-k_{3}^{*}\right)^{2} k_{1}^{2} k_{1}^{*}}{\left(k_{1}-k_{1}^{*}\right)^{2}\left(k_{1}-k_{2}^{*}\right)^{2}\left(k_{1}-k_{3}^{*}\right)^{2}\left(k_{2}-k_{1}^{*}\right)^{2}\left(k_{3}-k_{1}^{*}\right)^{2}} .
\end{aligned}
$$

For fixed $\eta_{2}$, note that $\eta_{1}+\eta_{1}^{*}=2 \operatorname{Re}\left(\frac{k_{1}}{k_{2}} \eta_{2}\right)+2 \operatorname{Re}\left(\frac{k_{1}}{k_{2}} \omega_{2}-\omega_{1}\right) t$ and $\eta_{3}+\eta_{3}^{*}=2 \operatorname{Re}\left(\frac{k_{3}}{k_{2}} \eta_{2}\right)+$ $2 \operatorname{Re}\left(\frac{k_{3}}{k_{2}} \omega_{2}-\omega_{3}\right) t$. Supposing $\operatorname{Re}\left(\frac{k_{3}}{k_{2}} \omega_{2}-\omega_{3}\right)>0$, it is obvious that $\operatorname{Re}\left(\frac{k_{1}}{k_{2}} \omega_{2}-\omega_{1}\right)<0$.

i) Taking limit $t \rightarrow-\infty: \eta_{1}+\eta_{1}^{*} \sim+\infty, \eta_{2}+\eta_{2}^{*} \sim 0, \eta_{3}+\eta_{3}^{*} \sim-\infty$, the asymptotic expressions for the three solitons before interaction can be given by

$$
\begin{aligned}
& u^{2-} \sim \frac{B_{1}}{2 A_{1}} e^{\frac{\eta_{2}-\eta_{2}^{*}-\alpha_{6}}{2}} \operatorname{sech}\left(\frac{\eta_{2}+\eta_{2}^{*}+\alpha_{6}}{2}\right), \\
& e^{\alpha_{6}}=\frac{C_{1}}{A_{1}}=-\frac{\left(k_{1}-k_{2}\right)^{2}\left(k_{1}^{*}-k_{2}^{*}\right)^{2} k_{2}^{2} k_{2}^{*}}{\left(k_{1}-k_{2}^{*}\right)^{2}\left(k_{2}-k_{1}^{*}\right)^{2}\left(k_{2}-k_{2}^{*}\right)^{2}} .
\end{aligned}
$$

ii) Taking limit $t \rightarrow+\infty: \eta_{1}+\eta_{1}^{*} \sim-\infty, \eta_{2}+\eta_{2}^{*} \sim 0, \eta_{3}+\eta_{3}^{*} \sim+\infty$, the asymptotic expressions for the three solitons after interaction can be given by

$$
\begin{aligned}
& u^{2+} \sim \frac{B_{9}}{2 A_{9}} e^{\frac{\eta_{2}-\eta_{2}^{*}-\alpha_{7}}{2}} \operatorname{sech}\left(\frac{\eta_{2}+\eta_{2}^{*}+\alpha_{7}}{2}\right), \\
& e^{\alpha_{7}}=\frac{C_{9}}{A_{9}}=-\frac{\left(k_{2}-k_{3}\right)^{2}\left(k_{2}^{*}-k_{3}^{*}\right)^{2} k_{2}^{2} k_{2}^{*}}{\left(k_{2}-k_{2}^{*}\right)^{2}\left(k_{2}-k_{3}^{*}\right)^{2}\left(k_{3}-k_{2}^{*}\right)^{2}} .
\end{aligned}
$$

For fixed $\eta_{3}$, note that $\eta_{1}+\eta_{1}^{*}=2 \operatorname{Re}\left(\frac{k_{1}}{k_{3}} \eta_{3}\right)+2 \operatorname{Re}\left(\frac{k_{1}}{k_{3}} \omega_{3}-\omega_{1}\right) t$ and $\eta_{2}+\eta_{2}^{*}=2 \operatorname{Re}\left(\frac{k_{2}}{k_{3}} \eta_{3}\right)+$ $2 \operatorname{Re}\left(\frac{k_{2}}{k_{3}} \omega_{3}-\omega_{2}\right) t$. It is obvious that $\operatorname{Re}\left(\frac{k_{1}}{k_{3}} \omega_{3}-\omega_{1}\right)<0$ and $\operatorname{Re}\left(\frac{k_{2}}{k_{3}} \omega_{3}-\omega_{2}\right)<0$. 
i) Taking limit $t \rightarrow-\infty: \eta_{1}+\eta_{1}^{*} \sim+\infty, \eta_{2}+\eta_{2}^{*} \sim+\infty, \eta_{3}+\eta_{3}^{*} \sim 0$, the asymptotic expressions for the three solitons before interaction can be given by

$$
\begin{aligned}
u^{3-} & \sim \frac{D_{1}}{2 C_{1}} e^{\frac{\eta_{3}-\eta_{3}^{*}-\alpha_{8}}{2}} \operatorname{sech}\left(\frac{\eta_{3}+\eta_{3}^{*}+\alpha_{8}}{2}\right), \\
e^{\alpha_{8}} & =\frac{E_{1}}{C_{1}}=-\frac{\left(k_{1}-k_{3}\right)^{2}\left(k_{1}^{*}-k_{3}^{*}\right)^{2}\left(k_{2}-k_{3}\right)^{2}\left(k_{2}^{*}-k_{3}^{*}\right)^{2} k_{3}^{2} k_{3}^{*}}{\left(k_{1}-k_{3}^{*}\right)^{2}\left(k_{2}-k_{3}^{*}\right)^{2}\left(k_{3}-k_{1}^{*}\right)^{2}\left(k_{3}-k_{2}^{*}\right)^{2}\left(k_{3}-k_{3}^{*}\right)^{2}} .
\end{aligned}
$$

ii) Taking limit $t \rightarrow+\infty: \eta_{1}+\eta_{1}^{*} \sim-\infty, \eta_{2}+\eta_{2}^{*} \sim-\infty, \eta_{3}+\eta_{3}^{*} \sim 0$, the asymptotic expressions for the three solitons after interaction can be given by

$$
\begin{aligned}
u^{3+} & \sim \frac{1}{2} e^{\frac{\eta_{3}-\eta_{3}^{*}-\alpha_{9}}{2}} \operatorname{sech}\left(\frac{\eta_{3}+\eta_{3}^{*}+\alpha_{9}}{2}\right), \\
e^{\alpha_{9}} & =A_{9}=\frac{i k_{3}}{\left(k_{3}-k_{3}^{*}\right)\left(-\omega_{3}-\omega_{3}^{*}\right)} .
\end{aligned}
$$

Comparing the asymptotic expressions of three-soliton solution between before interaction and after interaction, we find that $k_{1}, k_{1}^{*}, k_{2}, k_{2}^{*}, k_{3}$ and $k_{3}^{*}$ accord with the conditions

$$
\begin{aligned}
& \frac{k_{2}^{2} k_{3}^{2}\left|k_{1}-k_{2}^{*}\right|\left|k_{1}-k_{3}^{*}\right|\left|k_{1}^{*}-k_{2}^{*}\right|\left|k_{1}^{*}-k_{3}^{*}\right|}{k_{2}^{* 2} k_{3}^{* 2}\left|k_{1}-k_{2}\right|\left|k_{1}-k_{3}\right|\left|k_{2}-k_{1}^{*}\right|\left|k_{3}-k_{1}^{*}\right|}=1, \\
& \frac{k_{1}^{2} k_{3}^{* 2}\left|k_{2}-k_{3}\right|\left|k_{3}-k_{2}^{*}\right|\left|k_{2}-k_{1}^{*}\right|\left|k_{1}^{*}-k_{2}^{*}\right|}{k_{1}^{* 2} k_{3}^{2}\left|k_{1}-k_{2}\right|\left|k_{1}-k_{2}^{*}\right|\left|k_{2}-k_{3}^{*}\right|\left|k_{2}^{*}-k_{3}^{*}\right|}=1, \\
& \frac{k_{1}^{2} k_{2}^{2}\left|k_{1}^{*}-k_{3}^{*}\right|\left|k_{2}^{*}-k_{3}^{*}\right|\left|k_{3}-k_{1}^{*}\right|\left|k_{3}-k_{2}^{*}\right|}{k_{1}^{* 2} k_{2}^{* 2}\left|k_{1}-k_{3}\right|\left|k_{1}-k_{3}^{*}\right|\left|k_{2}-k_{3}\right|\left|k_{2}-k_{3}^{*}\right|}=1,
\end{aligned}
$$

the relations of amplitudes can be obtained

$$
\mathrm{Am}^{1-}=\mathrm{Am}^{1+}, \quad \mathrm{Am}^{2-}=\mathrm{Am}^{2+} \text { and } \mathrm{Am}^{3-}=\mathrm{Am}^{3+},
$$

where $\mathrm{Am}^{1-}, \mathrm{Am}^{2-}$ and $\mathrm{Am}^{3-}$ denote the amplitudes for the three solitons before the interaction, while $\mathrm{Am}^{1+}, \mathrm{Am}^{2+}$ and $\mathrm{Am}^{3+}$ denote the amplitudes for the three solitons after the interaction. When $k_{1}, k_{1}^{*}, k_{2}, k_{2}^{*}, k_{3}$ and $k_{3}^{*}$ do not accord with conditions (84)-(86), we have

$$
\mathrm{Am}^{1-} \neq \mathrm{Am}^{1+}, \quad \mathrm{Am}^{2-} \neq \mathrm{Am}^{2+} \text { and } \mathrm{Am}^{3-} \neq \mathrm{Am}^{3+} .
$$

Through expressions (87) and (88), it is obvious that the elastic interaction for three-soliton of the reverse space nonlocal FL equation appears under conditions (84)-(86), inelastic interaction for three-soliton of the reverse space nonlocal FL equation arises beyond conditions (84)-(86).

\section{Lax pair and conservation laws for three types of non- local FL equations}

\subsection{Lax pair and integrability}

In this subsection, the integrability of nonlocal FL equations will be shown by finding their Lax pairs which constructed from matrix generalization. The Lax pair for the reverse space nonlocal 
FL equation (1) can be expressed as follows

$$
\Psi_{S, x}=U_{1} \Psi_{S}, \quad \Psi_{S, t}=V_{1} \Psi_{S}
$$

with

$$
\begin{aligned}
U_{1} & =\left(\begin{array}{cc}
\frac{1}{2} \lambda^{2} & -\lambda u_{x}(x, t) \\
\lambda u_{x}^{*}(-x, t) & -\frac{1}{2} \lambda^{2}
\end{array}\right), \\
V_{1} & =\left(\begin{array}{cc}
\frac{i}{2 \lambda^{2}}-i u(x, t) u^{*}(-x, t) & \frac{i}{\lambda} u(x, t) \\
\frac{i}{\lambda} u^{*}(-x, t) & -\frac{i}{2 \lambda^{2}}+i u(x, t) u^{*}(-x, t)
\end{array}\right),
\end{aligned}
$$

where $\Psi_{S}=\left(\psi_{S, 1}, \psi_{S, 2}\right)^{T}$ is a column vector function, and $\Psi_{T}$ and $\Psi_{S T}$ below are also column vector functions. The compatibility condition of the Lax pair, which is zero curvature equation $U_{1 t}-V_{1 x}+\left[U_{1}, V_{1}\right]=0$, leads to Eq.(1). These variable transformations (2) and (3) allow us to derive the Lax pair of the reverse time and reverse space-time nonlocal FL equations from that of the reverse space one. The Lax pair for the reverse time nonlocal FL equation (4) is derived as follows

$$
\Psi_{T, x}=U_{2} \Psi_{T}, \quad \Psi_{T, t}=V_{2} \Psi_{T}
$$

with

$$
\begin{aligned}
U_{2} & =\left(\begin{array}{cc}
-\frac{i}{2} \lambda^{2} & -\lambda u_{x}(x, t) \\
\lambda u_{x}^{*}(x,-t) & \frac{i}{2} \lambda^{2}
\end{array}\right), \\
V_{2}= & \left(\begin{array}{cc}
-\frac{1}{2 \lambda^{2}}+u(x, t) u^{*}(x,-t) & -\frac{1}{\lambda} u(x, t) \\
-\frac{1}{\lambda} u^{*}(x,-t) & \frac{1}{2 \lambda^{2}}-u(x, t) u^{*}(x,-t)
\end{array}\right) .
\end{aligned}
$$

The Lax pair for the reverse space-time nonlocal FL equation (5) is shown as follows

$$
\Psi_{S T, x}=U_{3} \Psi_{S T}, \quad \Psi_{S T, t}=V_{3} \Psi_{S T}
$$

with

$$
\begin{aligned}
& U_{3}=\left(\begin{array}{cc}
-\frac{1}{2} \lambda^{2} & -\lambda u_{x}(x, t) \\
\lambda u_{x}^{*}(-x,-t) & \frac{1}{2} \lambda^{2}
\end{array}\right), \\
& V_{3}=\left(\begin{array}{cc}
-\frac{1}{2 \lambda^{2}}+u(x, t) u^{*}(-x,-t) & -\frac{1}{\lambda} u(x, t) \\
-\frac{1}{\lambda} u^{*}(-x,-t) & \frac{1}{2 \lambda^{2}}-u(x, t) u^{*}(-x,-t)
\end{array}\right) .
\end{aligned}
$$

The transformation relationship between these equations provides an effective method for us to derive the Lax pairs of different equations. In fact, given the solutions of the reverse space nonlocal FL equation, the solutions of reverse time and reverse space-time counterparts can be derived from the principle. However, if not, then the solutions of reverse time and reverses pace-time nonlocal FL equation may be derive desired solutions by other methods. 


\subsection{Conservation laws}

Based on the Lax pair, the infinitely many conservation laws are constructed in both positive and negative orders. We consider the associated spectral problem of the reverse space nonlocal FL equation

$$
\left(\begin{array}{c}
\psi_{1} \\
\psi_{2}
\end{array}\right)_{x}=\left(\begin{array}{cc}
\frac{1}{2} \lambda^{2} & -\lambda u_{x} \\
\lambda u_{x}^{*} & -\frac{1}{2} \lambda^{2}
\end{array}\right)\left(\begin{array}{c}
\psi_{1} \\
\psi_{2}
\end{array}\right)
$$

and associate time evolution equation

$$
\left(\begin{array}{c}
\psi_{1} \\
\psi_{2}
\end{array}\right)_{t}=\left(\begin{array}{cc}
\frac{i}{2 \lambda^{2}}-i u u^{*} & \frac{i}{\lambda} u \\
\frac{i}{\lambda} u^{*} & -\frac{i}{2 \lambda^{2}}+i u u^{*}
\end{array}\right)\left(\begin{array}{l}
\psi_{1} \\
\psi_{2}
\end{array}\right) .
$$

They satisfy the following expression

$$
\begin{aligned}
& u_{x}\left(\frac{\psi_{2}}{\psi_{1}}\right)_{x}=\lambda u_{x} u_{x}^{*}-\lambda^{2} u_{x} \frac{\psi_{2}}{\psi_{1}}+\lambda u_{x}^{2}\left(\frac{\psi_{2}}{\psi_{1}}\right)^{2} \\
& u\left(\frac{\psi_{2}}{\psi_{1}}\right)_{t}=\frac{i}{\lambda} u u^{*}+\left(-\frac{i}{\lambda^{2}}+2 i u u^{*}\right) u \frac{\psi_{2}}{\psi_{1}}-\frac{i}{\lambda} u^{2}\left(\frac{\psi_{2}}{\psi_{1}}\right)^{2}
\end{aligned}
$$

The expression of $\frac{\psi_{2}}{\psi_{1}}$ is given as follows

$$
\frac{\psi_{2}}{\psi_{1}}=\sum_{i=1}^{\infty} \mathbf{P}_{i} \lambda^{-2 i+1}
$$

Substituting (96) into Eq. (94), and comparing the coefficients of $\lambda$, we obtain

$$
\begin{aligned}
& P_{1}=u_{x}^{*} \\
& P_{i+1}=-P_{i, x}+\sum_{j=1}^{i} u_{x} P_{j} P_{i+1-j} \cdot(i=1,2, \ldots)
\end{aligned}
$$

It can be easily shown that $\psi_{1}$ satisfies

$$
\left(\ln \psi_{1}\right)_{x t}=\left(\ln \psi_{1}\right)_{t x}
$$

Hence, the conservation laws are derived as follows

$$
\left(-\lambda u_{x} \frac{\psi_{2}}{\psi_{1}}\right)_{t}=\left(-i u u^{*}+i \frac{1}{\lambda} u \frac{\psi_{2}}{\psi_{1}}\right)_{x}
$$

which can be written as

$$
\begin{aligned}
& \left(u_{x} P_{i}\right)_{t}=-\left(i u P_{i-1}\right)_{x},(i=1,2, \ldots), \\
& P_{0}=-u^{*}
\end{aligned}
$$


Among thses conservation laws, the first two are listed below

$$
\begin{aligned}
& \left(u_{x} u_{x}^{*}\right)_{t}=\left(i u u^{*}\right)_{x}, \\
& {\left[u_{x}\left(-u_{x x}^{*}+u_{x} u_{x}^{* 2}\right)\right]_{t}=\left(-i u u_{x}^{*}\right)_{x} .}
\end{aligned}
$$

On the other hand, substituting the expansion

$$
\frac{\psi_{2}}{\psi_{1}}=\sum_{i=1}^{\infty} \mathbf{Q}_{i} \lambda^{2 i-1}
$$

into Eq. (95) and comparing the coefficients of $\lambda$, one obtains

$$
\begin{aligned}
& Q_{1}=u^{*}, \\
& Q_{i+1}=i Q_{i, t}+2 u u^{*} Q_{i}-\sum_{j=1}^{i} u P_{j} P_{i+1-j} \quad(i=1,2, \ldots) .
\end{aligned}
$$

Then other conservation laws are given as follows

$$
\left(u_{x} Q_{i}\right)_{t}=-i\left(u P_{i+1}\right)_{x} \quad(i=1,2, \ldots) .
$$

Among thses conservation laws, the first two are listed below

$$
\begin{aligned}
& \left(u_{x} u^{*}\right)_{t}=-i\left[u\left(i u_{t}^{*}+u u^{* 2}\right)\right]_{x}, \\
& {\left[u_{x}\left(i u_{t}^{*}+u u^{* 2}\right)\right]_{t}=-i\left[u\left(-u_{t t}^{*}+i\left(u u^{* 2}\right)_{t}\right)\right]_{x} .}
\end{aligned}
$$

The transformations Eqs.(2)-(3) allows us to derive the conversation laws of the reverse time and reverse space-time nonlocal FL equation from those of the reverse space ones. The first two conversation laws for the reverse time nonlocal FL equation (4) are derived as follows

$$
\begin{aligned}
& \left(-i u_{x} u_{x}^{*}\right)_{t}=\left(u u^{*}\right)_{x}, \\
& {\left[u_{x}\left(u_{x x}-i u_{x} u_{x}^{* 2}\right)\right]_{t}=\left(i u u_{x}^{*}\right)_{x},}
\end{aligned}
$$

and

$$
\begin{aligned}
& \left(u_{x} u^{*}\right)_{t}=\left[u\left(u_{t}^{*}+u u^{* 2}\right)\right]_{x}, \\
& {\left[u_{x}\left(u_{t}^{*}+u u^{* 2}\right)\right]_{t}=\left[u\left(u_{t t}^{*}+\left(u u^{* 2}\right)_{t}\right)\right]_{x} .}
\end{aligned}
$$

The first two conversation laws for the reverse space-time nonlocal FL equation (5) are derived as follows

$$
\begin{aligned}
& \left(u_{x} u_{x}^{*}\right)_{t}=\left(u u^{*}\right)_{x}, \\
& {\left[u_{x}\left(u_{x x}^{*}+u_{x} u_{x}^{* 2}\right)\right]_{t}=\left(u u_{x}^{*}\right)_{x},}
\end{aligned}
$$


and

$$
\begin{aligned}
& \left(u_{x} u^{*}\right)_{t}=\left[u\left(u_{t}^{*}+u u^{* 2}\right)\right]_{x} \\
& {\left[u_{x}\left(u_{t}^{*}+u u^{* 2}\right)\right]_{t}=\left[u\left(u_{t t}^{*}+\left(u u^{* 2}\right)_{t}\right)\right]_{x} .}
\end{aligned}
$$

So, through the transformation relationship between these equations, it is effective to provide the conversation laws of different equations. However the prerequisite for doing these things is knowing the Lax pairs of these equations.

\section{Conclusions}

In this paper, three types of nonlocal Fokas-Lenells equations are considered by means of the Hirota bilinear method. The one-, two- and three-soliton solutions of the reverse time and reverse space-time nonlocal FL equation are converted from those of the reverse space ones. Furthermore, the graphical representations are presented showing the shape of solution more visually, and the physical interpretation of the obtained figures is discussed for different choices of the parameters that occur in the solutions. Then, asymptotic analysis of two- and three-soliton solutions of reverse space nonlocal FL equation are given to understand the long time asymptotic behavior. The Lax integrability of three types of nonlocal FL equations is investigated using variable transformations, and infinitely many conservation laws are constructed based on the Lax pairs of different equations. These results might be useful to comprehend some physical phenomena and inspire some novel physical applications on other nonlinear system.

\section{Acknowledgments}

This work is supported by the National Natural Science Foundation of China (Nos. 11905013 and 11772063), Qin Xin Talents Cultivation Program of Beijing Information Science and Technology University (QXTCP C202118), the Scientific Research Common Program of Beijing Municipal Commission of Education under Grant (No. KM201911232011).

\section{Compliance with ethical standards}

Data availability statement All data generated or analysed during this study are included in this published article.

Conflict of interest The authors declare that they have no conflict of interest. 


\section{References}

[1] A. S. Fokas, On a class of physically important integrable equations, Phys. D 87 (1995) $145-150$.

[2] J. Lenells and A. S. Fokas, On a novel integrable generalization of the nonlinear Schrödinger equation, Nonlinearity. 22 (2008) 11-27.

[3] J. Lenells, Exactly solvable model for nonlinear pulse propagation in optical fibers, Stud. Appl. Math. 123 (2009) 215-232.

[4] M. Arshad, D. Lu, M. U. Rehman, I. Ahmed and A. M. Sultan, Optical solitary wave and elliptic function solutions of the Fokas-Lenells equation in the presence of perturbation terms and its modulation instability, Phys. Scr. 94 (2019) 105202.

[5] M. Wang and Y. Chen, Dynamic behaviors of mixed localized solutions for the threecomponent coupled Fokas-Lenells system, Nonlinear Dyn. 98 (2019) 1781-1794.

[6] H. Triki and A. M. Wazwaz, Combined optical solitary waves of the Fokas-Lenells equation, Wave Random Complex 27 (2017) 587-593.

[7] P. Zhao, E. Fan, Y. Hou, Algebro-geometric solutions and their reductions for the FokasLenells hierarchy, J. Nonlinear Math. Phys. 20 (2013) 355-393.

[8] L. Ling, B. F. Feng and Z. Zhu, General soliton solutions to a coupled Fokas-Lenells equation, Nonlinear Anal. Real 40 185-214.

[9] S. Xu, J. He, Y. Cheng and K. Porseizan, The n-order rogue waves of Fokas-Lenells equation, Math. Meth. Appl. 38 (2015) 1106-1126.

[10] Q. Zhang, Y. Zhang and R. Ye, Exact solutions of nonlocal Fokas-Lenells equation, Appl. Math. Lett. 98 (2019) 336.

[11] F. Liu, C. C. Zhou, X. Lü and H. Xu, Dynamic behaviors of optical solitons for Fokas-Lenells equation in optical fiber, Optik 224 (2020) 165237.

[12] Y. Matsuno, A direct method of solution for the Fokas-Lenells derivative nonlinear Schrödinger equation: I. Bright soliton solutions, J. Phys. A: Math. Theor. 45 (2012) 235202.

[13] Y. Matsuno, A direct method of solution for the Fokas-Lenells derivative nonlinear Schrödinger equation: II. Dark soliton solutions, J. Phys. A: Math. Theor. 45 (2012) 475202.

[14] J. Xu and E. Fan, Long-time asymptotics for the Fokas-Lenells equation with decaying initial value problem: Without solitons, J. Differ. Equ. 259 (2015) 1098-1148. 
[15] Y. Zhao and E. Fan, Inverse scattering transformation for the Fokas-Lenells equation with nonzero boundary, 2019, arXiv:1912.12400v1.

[16] Z. Z. Kang, T. C. Xia and X. Ma, Multi-soliton solutions for the coupled Fokas-Lenells system via Riemann-Hilbert approach, China. Phys. Lett. 35 (2018) 070201.

[17] V. E. Vekslerchik, Lattice representation and dark solitons of the Fokas-Lenells equation, Nonlinearity. 24 (2011) 1165-1175.

[18] H. Triki and A. M. Wazwaz, New types of chirped soliton solutions for the Fokas-Lenells equation, Int. J. Numer. Method. H. 27 (2017) 1596-1601.

[19] M. J. Ablowitz and Z. H. Musslimani, Integrable nonlocal nonlinear Schrödinger equation, Phys. Rev. Lett. 110 (2013) 064105.

[20] B. Yang and J. Yang, Transformations between nonlocal and local integrable equations, Stud. Appl. Math. 140 (2017) 178-201.

[21] M. J. Ablowitz and Z. H. Musslimani, Integrable nonlocal nonlinear equations, Stud. Appl. Math. 139 (2016) 7-59.

[22] B. Wang, Z. Zhang and B. Li, Two types of smooth positons for nonlocal Fokas-Lenells equation, Int. J. Mod. Phys. B, (2020) 2050148 (9 pages).

[23] M. Gürses and A. Pekcan, Nonlocal nonlinear Schrödinger equations and their soliton solutions, J. Math. Phys. 59 (2018) 051501.

[24] M. Gürses and A. Pekcan, Nonlocal modified KdV equations and their soliton solutions by Hirota method, Commun. Nonlinear. 67 (2019) 427-448.

[25] Y. Yang, T. Suzuki, X. Cheng, Darboux transformations and exact solutions for the integrable nonlocal Lakshmanan-Porsezian-Daniel equation, Appl. Math. Lett. 99 (2020) 105998.

[26] F.J He, E.G. Fan and J. Xu, Long-Time asymptotics for the nonlocal mKdV equation, Commun. Theor. Phys. 71 (2019) 475-488.

[27] B.F. Feng, X.D. Luo, M. J Ablowitz, and Z. H Musslimani, General soliton solution to a nonlocal nonlinear Schrödinger equation with zero and nonzero boundary conditions, Nonlinearity 31 (2018) 5385-5409.

[28] W.Q. Peng, S.F. Tian, T,T, Zhang, Y. Fang, Rational and semi-rational solutions of a nonlocal (2+1)-dimensional nonlinear Schrödinger equation, Math. Meth. Appl. Sci. (2019) 1-13. 
[29] S. Liu, H. Wu, D.J. Zhang, New dynamics of the classical and nonlocal Gross-Pitaevskii equation with a parabolic potential, Rep. Math. Phys., 86 (2020) 271-292.

[30] R. Hirota, The Direct Method in Soliton Theory, Cambridge University Press, New York, 2004.

[31] W. J. Liu, B. Tian, H. Q. Zhang, L. L. Li and Y. S. Xue, Soliton interaction in the higherorder nonlinear Schrödinger equation investigated with Hirota-bilinear method, Phys. Rev. E 77 (2008) 066605.

[32] Y. Zhang, S. F. Deng, D. J. Zhang and D. Y. Chen, The N-soliton solutions for the nonisospectral mKdV equation, Phys. A 339 (2004) 228-236.

[33] W. X. Ma, X. Yong and H. Q. Zhang, Diversity of interaction solutions to the $(2+1)$ dimensional Ito equation, Comput. Math. Appl. 75 (2018) 289-295.

[34] T. Kanna and M. Lakshmanan, Exact soliton solutions of coupled nonlinear Schrödinger equations: Shape-changing collisions, logic gates, and partially coherent solitons, Phys. Rev. E 67 (2003) 046617.

[35] Y. Jiang, B. Tian, W. J. Liu, K. Sun, M. Li and P. Wang, Soliton interactions and complexes for coupled nonlinear Schrödinger equations, Phys. Rev. E 85 (2012) 036605. 